\title{
Thionylchloride catalyzed aldol condensation: Synthesis, spectral correlation and antibacterial activities of some 3,5-dichloro-2-hydroxyphenyl chalcones
}

\author{
R. Arulkumaran ${ }^{1}$, S. Vijayakumar ${ }^{1}$, R. Sundararajan ${ }^{1}$, S P. Sakthinathan ${ }^{1}$, \\ D. Kamalakkannan ${ }^{1}$, R. Suresh ${ }^{1}$, K. Ranganathan ${ }^{1}$, \\ G. Vanangamudi ${ }^{1}$, G. Thirunarayanan ${ }^{2, *}$ \\ ${ }^{1}$ PG \& Research Department of Chemistry, Government Arts College, C-Mutlur, \\ Chidambaram-608102, India. \\ ${ }^{2}$ Department of Chemistry, Annamalai University, Annamalainagar-608002, India. \\ *E-mail address: drgtnarayanan@gmail.com
}

\begin{abstract}
A series of substituted styryl 3,5-dichloro-2-hydroxyphenyl ketones [1-(3, 5-dichloro-2hydroxy)-3-phenylprop-2-en-1-one] were synthesized using thionyl chloride assisted Crossed-Aldol reaction. The yields of chalcones were more than $80 \%$. The synthesized chalcones were characterized by analytical and spectroscopic data. From the spectroscopic data the group frequencies were correlated with Hammett substituent constants, F and R parameters. From the results of statistical analysis the effect of substituents were discussed. The antibacterial activities of these chalcones have been evaluated using Bauer-Kirby method.
\end{abstract}

\section{Keywords:}

Thionylchloride; Styryl 3,5-dichloro-2-hydroxyphenyl ketones; IR spectra, NMR spectra; Hammett correlation; Substituent effects; Antimicrobial activities

\section{INTRODUCTION}

Chalcones are $\alpha, \beta$ unsaturated ketones possess methylene structural moieties and they belongs to biomolecules. Many alkyl-alkyl, alkyl-aryl and aryl-aryl categories of chalcones have been synthesized [1] and extracted from natural plants [2] by organic chemists. Various methods available for synthesizing chalcones such as Aldol, Crossed-Aldol, Claisen-Schmidt, Knovenagal, Greener methods-Grinding of reactants, solvent free and oxides of nanoparticles with microwave heating. Also microwave assisted solvent free Aldol and Crossed-Aldol condensation [3-5] were useful synthesis of carbonyl compounds. Due to C-C single bond rotation [6] of carbonyl and alkene carbons, they exist as $E s$-cis and $s$-trans and $Z s$-cis and $Z$ $s$-trans conformers. These structural conformers of chalcones have been confirmed by NMR and IR spectroscopy.

Chalcones possess various biological activities such as antibacterial $[7,8]$, antifungal [9], antioxidant [10], antiviral [11], antimalarial [12], antiplasmodial [13], antituberclosis 
[14], antiproliferative [15], antileshmanial [16], anti-inflammatory [17], antianalgesic and sedative [18], insect, antifeedants [19]. Halogenated chalcones possess insect antifeedant activities [20]. The presence of a reactive $\alpha, \beta$-unsaturated keto function in chalcones was found to be responsible for their antimicrobial activity. At present, scientists have paid more interest to correlate the group frequencies of spectral data with Hammett substituent constants to explain the substituent effects of organic compounds. There is no information available in the literature regarding Thionylchloride catalyzed aldol condensation between aryl ketones and aldehydes, spectral and antimicrobial activities. Therefore the authors have taken efforts for synthesizing some chalcones by thionylchloride assisted Aldol condensation, studied the spectral correlation and antimicrobial activities.

\section{EXPERIMENTAL}

\subsection{General}

All chemicals used were purchased from Sigma-Aldrich chemical company Bangalore. Melting points of all chalcones have been determined in open glass capillaries on Suntex melting point apparatus and are uncorrected. The ultra violet spectra of the chalcones synthesized have been recorded using ELICO-double beam BL222 Bio- Spectrophotometer. Infrared spectra $\left(\mathrm{KBr}, 4000-400 \mathrm{~cm}^{-1}\right)$ have been recorded on AVATAR-300 Fourier transform spectrophotometer. BRUKER-500MHz NMR spectrometers have been utilized for recording ${ }^{1} \mathrm{H}$ and ${ }^{13} \mathrm{C}$ spectra in $\mathrm{CDCl}_{3}$ solvent using TMS as internal standard.

\subsection{Synthesis of chalcones}

Appropriate mixture of 3,5-dichloro-2-hydroxyacetophenone $(100 \mathrm{mmol})$ and substituted benzaldehydes (100 mmol), $15 \mathrm{~mL}$ of diethyl ether and $(100 \mathrm{mmol})$ of thionylchloride were added. The reaction mixture was vigorously stirred at room temperature for 30 minutes (Scheme 1). After complete conversion of the ketones as monitored by TLC, the mixture was allowed to stand 20 minutes. The reagents were removed by filtration. The filtrate was washed with distilled water and recrystallized from absolute ethanol, dried well and kept in a desiccator.

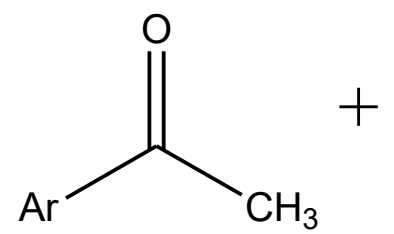

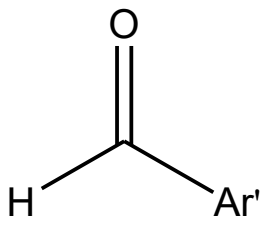

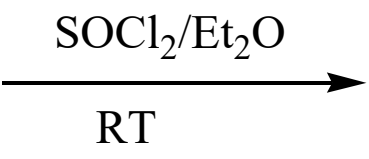<smiles>O=C(Br)C=CCBr</smiles>

1-38

Scheme 1. Thionyl chloride catalyzed aldol condensation between 3,5-dichloro-2hydroxyacetophenone and benzldehydes (entries 27-36). 


\section{RESULTS AND DISCUSSION}

In our organic chemistry research laboratory, we attempts to synthesize aryl chalcone derivatives by Crossed-Aldol condensation of electron withdrawing as well as electron donating group substituted aryl methyl ketones and benzaldehydes in the presence of vigorous acidic catalyst thionyl chloride in diethyl ether except acid or base or its salt in atmospheric temperature condition. Hence the authors have synthesized the chalcone derivatives by the reaction between $100 \mathrm{mmol}$ of aryl methyl ketones $100 \mathrm{mmol}$ substituted benzaldehydes, $100 \mathrm{mmol}$ of thionyl chloride and $15 \mathrm{~mL}$ of ether at room temperature (Scheme 1). During the course of this reaction the acidic thionyl chloride catalyzes Aldol reaction between aryl ketone and aldehydes and elimination of water gave the chalcones. The yields of the chalcones in this reaction are more than $80 \%$. The proposed general mechanism of this reaction is given in Fig. 1. Further we investigated this reaction with equimolar quantities of the 3,5-dichloro-2-hydroxyacetophenone and benzaldehyde (entry 27). In this reaction the obtained yield is $83 \%$. The physical constants yield and mass spectral data are presented in Table 1. We have studied the effect of solvent for this Aldol condensation by observing the yield of the products. The solvents such as ethanol, methanol, dichloromethane, dimethyl formamide and water have been used for this Aldol reaction with 3,5-dichloro-2hydroxyacetophenone and benzaldehyde. Carrying out this Aldol reaction with above solvents the resulting yields are $73 \%, 68 \%, 65 \%, 66 \%$ and $65 \%$ of chalcones respectively. The same reaction was carried out with reflux conditions and there is no improvement for the yield of the products. Here the authors have achieved the aim of this synthetic method with the observation of more than $82 \%$ yields of aryl chalcones by condensation of 3,5-dichloro2-hydroxyacetophenone and benzaldehyde in presence of $\mathrm{SOCl}_{2} / \mathrm{Et}_{2} \mathrm{O}$ in room temperature.<smiles>CCOC(C)(C)C(=O)c1cc(Cl)cc(Cl)c1O</smiles><smiles>[X]c1ccc(C(=O)c2ccc(C)cc2)cc1C(=C)OOC([R])(C)O[Na]</smiles><smiles>[X]c1ccc(C2=CC(c3cc(Cl)cc(Cl)c3O)=CC(OC)O2)cc1</smiles><smiles>[X]c1ccc(C(O)/C=C/c2cc(/C=C/C(=O)c3cc(Cl)cc(Cl)c3O)ccc2[X])cc1</smiles><smiles>[X]c1ccc(C2CC(c3cc(Cl)cc(Cl)c3O)OCO2)cc1</smiles>

Fig. 1. Proposed mechanism for the synthesis of substituted styryl 3,5-dichloro-2-hydroxyphenyl ketones by solfonyl chloride catalyzed Aldol reaction. 
Table 1. Analytical and mass spectral data of chalcones synthesized by $\mathrm{SOCl}_{2} / \mathrm{Et}_{2} \mathrm{O}$ catalyzed aryl methyl ketones and substituted benzaldehydes reaction of the type

$\mathrm{Ar}-\mathrm{CO}-\mathrm{CH}_{3}+\mathrm{Ar}^{\prime}-\mathrm{CHO} \rightarrow \mathrm{Ar}-\mathrm{CO}-\mathrm{CH}=\mathrm{CH}-\mathrm{Ar}^{\prime}$.

\begin{tabular}{|c|c|c|c|c|c|c|c|}
\hline Entry & Ar & Ar' & Product & $\begin{array}{l}\text { M. } \\
\text { W. }\end{array}$ & $\begin{array}{c}\text { Yiel } \\
\text { d } \\
(\%)\end{array}$ & $\begin{array}{l}\text { m.p. } \\
\left({ }^{\circ} \mathbf{C}\right)\end{array}$ & Mass \\
\hline 1 & $\mathrm{C}_{6} \mathrm{H}_{5}$ & $\mathrm{C}_{6} \mathrm{H}_{5}$ & $\mathrm{C}_{6} \mathrm{H}_{5} \mathrm{COCH}=\mathrm{CHC}_{6} \mathrm{H}_{5}$ & 208 & 81 & $\begin{array}{c}55-56 \\
(55- \\
58)[21]\end{array}$ & --- \\
\hline 2 & $4-\mathrm{N}\left(\mathrm{CH}_{3}\right)_{2} \mathrm{C}_{6} \mathrm{H}_{4}$ & $\mathrm{C}_{6} \mathrm{H}_{5}$ & $4-\mathrm{N}\left(\mathrm{CH}_{3}\right)_{2} \mathrm{C}_{6} \mathrm{H}_{4} \mathrm{COCH}=\mathrm{CHC}_{6} \mathrm{H}_{5}$ & 294 & 80 & $\begin{array}{c}87-88 \\
(87-88) \\
{[21]}\end{array}$ & --- \\
\hline 3 & $\mathrm{C}_{6} \mathrm{H}_{5}$ & $\mathrm{C}_{10} \mathrm{H}_{7}(1-\mathrm{Naph})$ & $\mathrm{C}_{6} \mathrm{H}_{5} \mathrm{COCH}=\mathrm{CHC}_{10} \mathrm{H}_{7}$ & 273 & 85 & $\begin{array}{c}104-105 \\
(104- \\
105)[21]\end{array}$ & --- \\
\hline 4 & $\mathrm{C}_{6} \mathrm{H}_{5}$ & $\mathrm{C}_{8} \mathrm{H}_{6} \mathrm{~N}(3-$-Ind $)$ & $\mathrm{C}_{6} \mathrm{H}_{5} \mathrm{COCH}=\mathrm{CHC}_{8} \mathrm{H}_{6} \mathrm{~N}$ & 262 & 80 & $\begin{array}{c}140-141 \\
(140)[21]\end{array}$ & --- \\
\hline 5 & $\mathrm{C}_{6} \mathrm{H}_{5}$ & $4-\mathrm{OHC}_{6} \mathrm{H}_{4}$ & $\mathrm{C}_{6} \mathrm{H}_{5} \mathrm{COCH}=\mathrm{CHC}_{6} \mathrm{H}_{4} \mathrm{OH}$ & 291 & 82 & $\begin{array}{c}122-123 \\
(122)[21]\end{array}$ & -- \\
\hline 6 & $\mathrm{C}_{6} \mathrm{H}_{5}$ & $4-\mathrm{OCH}_{3} \mathrm{C}_{6} \mathrm{H}_{4}$ & $\mathrm{C}_{6} \mathrm{H}_{5} \mathrm{COCH}=\mathrm{CHC}_{6} \mathrm{H}_{4} \mathrm{OCH}_{3}$ & 291 & 80 & $\begin{array}{l}164-165 \\
(164)[21]\end{array}$ & --- \\
\hline 7 & $4-\mathrm{NH}_{2} \mathrm{C}_{6} \mathrm{H}_{5}$ & $\mathrm{C}_{4} \mathrm{H}_{3}$ (3-Furyl) & $4-\mathrm{NH}_{2} \mathrm{C}_{6} \mathrm{H}_{5} \mathrm{COCH}=\mathrm{CHC}_{4} \mathrm{H}_{3}$ & 213 & 81 & $\begin{array}{c}120-121 \\
(119-120) \\
{[21]}\end{array}$ & --- \\
\hline 8 & $4-\mathrm{NH}_{2} \mathrm{C}_{6} \mathrm{H}_{4}$ & $\mathrm{C}_{6} \mathrm{H}_{5}$ & $4-\mathrm{NH}_{2} \mathrm{C}_{6} \mathrm{H}_{4} \mathrm{COCH}=\mathrm{CHC}_{6} \mathrm{H}_{5}$ & 223 & 82 & $\begin{array}{c}98-99 \\
(98-99) \\
{[21]}\end{array}$ & --- \\
\hline 9 & $4-\mathrm{NH}_{2} \mathrm{C}_{6} \mathrm{H}_{4}$ & $2,6-\mathrm{Cl}_{2} \mathrm{C}_{6} \mathrm{H}_{3}$ & $4-\mathrm{NH}_{2} \mathrm{C}_{6} \mathrm{H}_{4} \mathrm{COCH}=\mathrm{CHC}_{6} \mathrm{H}_{3} \mathrm{Cl}_{2}$ & 291 & 80 & $\begin{array}{c}98-99 \\
(98-99) \\
{[21]}\end{array}$ & --- \\
\hline 10 & $4-\mathrm{FC}_{6} \mathrm{H}_{4}$ & $\mathrm{C}_{6} \mathrm{H}_{5}$ & $4-\mathrm{FC}_{6} \mathrm{H}_{4} \mathrm{COCH}=\mathrm{CHC}_{6} \mathrm{H}_{5}$ & 226 & 81 & $\begin{array}{c}49-50 \\
(49-50) \\
{[21]}\end{array}$ & --- \\
\hline 11 & $2,4-\mathrm{Cl}_{2} \mathrm{C}_{6} \mathrm{H}_{3}$ & $\mathrm{C}_{6} \mathrm{H}_{5}$ & $2,4-\mathrm{Cl}_{2} \mathrm{C}_{6} \mathrm{H}_{3} \mathrm{COCH}=\mathrm{CHC}_{6} \mathrm{H}_{5}$ & 276 & 84 & $\begin{array}{c}80-82 \\
(80-81) \\
{[21]}\end{array}$ & \\
\hline 12 & $3,4-\mathrm{Cl}_{2} \mathrm{C}_{6} \mathrm{H}_{3}$ & $\mathrm{C}_{6} \mathrm{H}_{5}$ & $3,4-\mathrm{Cl}_{2} \mathrm{C}_{6} \mathrm{H}_{3} \mathrm{COCH}=\mathrm{CHC}_{6} \mathrm{H}_{5}$ & 276 & 82 & $\begin{array}{c}100-101 \\
(100-101) \\
{[21]}\end{array}$ & \\
\hline 13 & $4-(\mathrm{OH}) \mathrm{C}_{6} \mathrm{H}_{4}$ & $\mathrm{C}_{6} \mathrm{H}_{5}$ & $4-(\mathrm{OH}) \mathrm{C}_{6} \mathrm{H}_{3} \mathrm{COCH}=\mathrm{CHC}_{6} \mathrm{H}_{5}$ & 226 & 80 & $\begin{array}{c}63-64 \\
(63-64) \\
{[21]}\end{array}$ & \\
\hline 14 & $\mathrm{C}_{10} \mathrm{H}_{7}(1-\mathrm{Naph})$ & $\mathrm{C}_{6} \mathrm{H}_{5}$ & $\mathrm{C}_{10} \mathrm{H}_{7} \mathrm{COCH}=\mathrm{CHC}_{6} \mathrm{H}_{5}$ & 258 & 80 & $\begin{array}{c}100-102 \\
(100-102) \\
{[21]}\end{array}$ & \\
\hline 15 & $4-\mathrm{BrC}_{10} \mathrm{H}_{6}(1-\mathrm{Naph})$ & $\mathrm{C}_{6} \mathrm{H}_{5}$ & $4-\mathrm{BrC}_{10} \mathrm{H}_{6} \mathrm{COCH}=\mathrm{CHC}_{6} \mathrm{H}_{5}$ & 396 & 82 & $\begin{array}{c}103-104 \\
(103-104) \\
{[21]}\end{array}$ & \\
\hline 16 & $4-\mathrm{ClC}_{10} \mathrm{H}_{6}(1-\mathrm{Naph})$ & $\mathrm{C}_{6} \mathrm{H}_{5}$ & $4-\mathrm{ClC}_{10} \mathrm{H}_{6} \mathrm{COCH}=\mathrm{CHC}_{6} \mathrm{H}_{5}$ & 292 & 83 & $\begin{array}{c}122-123 \\
(122-123) \\
{[21]}\end{array}$ & \\
\hline 17 & $\mathrm{C}_{10} \mathrm{H}_{7}(2-\mathrm{Naph})$ & $\mathrm{C}_{6} \mathrm{H}_{5}$ & $\mathrm{C}_{10} \mathrm{H}_{7} \mathrm{COCH}=\mathrm{CHC}_{6} \mathrm{H}_{5}$ & 258 & 81 & $\begin{array}{c}104-105 \\
(104-105) \\
{[21]}\end{array}$ & \\
\hline 18 & $\mathrm{C}_{13} \mathrm{H}_{9}(2$-Fluorene $)$ & $\mathrm{C}_{6} \mathrm{H}_{5}$ & $\mathrm{C}_{13} \mathrm{H}_{9} \mathrm{COCH}=\mathrm{CHC}_{6} \mathrm{H}_{5}$ & 296 & 80 & $\begin{array}{c}150-151 \\
(150-151) \\
{[21]}\end{array}$ & --- \\
\hline
\end{tabular}




\begin{tabular}{|c|c|c|c|c|c|c|c|}
\hline 19 & $\mathrm{C}_{12} \mathrm{H}_{9}$ (Biphenyl) & $\mathrm{C}_{6} \mathrm{H}_{5}$ & $\mathrm{C}_{12} \mathrm{H}_{9} \mathrm{COCH}=\mathrm{CHC}_{6} \mathrm{H}_{5}$ & 284 & 83 & $\begin{array}{c}153-154 \\
(153- \\
154[21]\end{array}$ & --- \\
\hline 20 & $\mathrm{C}_{4} \mathrm{H}_{3} \mathrm{O}(2$-Furyl) & $\mathrm{C}_{6} \mathrm{H}_{5}$ & $\mathrm{C}_{4} \mathrm{H}_{3} \mathrm{OCOCH}=\mathrm{CHC}_{6} \mathrm{H}_{5}$ & 198 & 80 & $\begin{array}{c}80-81 \\
(80-81) \\
{[21]}\end{array}$ & --- \\
\hline 21 & $\mathrm{C}_{4} \mathrm{H}_{3} \mathrm{~S}$ (2-Thienyl) & $\mathrm{C}_{6} \mathrm{H}_{5}$ & $\mathrm{C}_{4} \mathrm{H}_{3} \mathrm{SCOCH}=\mathrm{CHC}_{6} \mathrm{H}_{5}$ & 204 & 83 & $\begin{array}{c}112-113 \\
(112-113) \\
{[21]}\end{array}$ & --- \\
\hline 22 & $\mathrm{C}_{14} \mathrm{H}_{10}($ Anthracene $)$ & $\mathrm{C}_{6} \mathrm{H}_{5}$ & $\mathrm{C}_{14} \mathrm{H}_{9} \mathrm{COCH}=\mathrm{CHC}_{6} \mathrm{H}_{5}$ & 308 & 82 & $\begin{array}{c}124-125 \\
(124-125) \\
{[21]}\end{array}$ & --- \\
\hline 23 & 5- $\mathrm{BrC}_{4} \mathrm{H}_{2} \mathrm{~S}(2-\mathrm{Thyl})$ & $\mathrm{C}_{6} \mathrm{H}_{5}$ & $5-\mathrm{BrC}_{4} \mathrm{H}_{2} \mathrm{SCOCH}=\mathrm{CHC}_{6} \mathrm{H}_{5}$ & 292 & 83 & $\begin{array}{c}107-110 \\
(107-110) \\
{[21]}\end{array}$ & --- \\
\hline 24 & 5- $\mathrm{BrC}_{4} \mathrm{H}_{2} \mathrm{~S}(2-\mathrm{Thyl})$ & 4- $\mathrm{BrC}_{6} \mathrm{H}_{4}$ & $5-\mathrm{BrC}_{4} \mathrm{H}_{2} \mathrm{SCOCH}=\mathrm{CHC}_{6} \mathrm{H}_{4} \mathrm{Br}$ & 371 & 82 & $\begin{array}{c}173-174 \\
(173-174) \\
{[21]}\end{array}$ & --- \\
\hline 25 & 4-Cyclohexylphenyl & $\mathrm{C}_{6} \mathrm{H}_{5}$ & $4-\mathrm{C}_{6} \mathrm{H}_{11} \mathrm{C}_{6} \mathrm{H}_{4} \mathrm{CO} \mathrm{CH}=\mathrm{CHC}_{6} \mathrm{H}_{5}$ & 290 & 83 & $\begin{array}{c}105-106 \\
(104-105) \\
{[21]}\end{array}$ & --- \\
\hline 26 & 4-Cyclohexylphenyl & $3-\mathrm{BrC}_{6} \mathrm{H}_{4}$ & $4-\mathrm{C}_{6} \mathrm{H}_{11} \mathrm{C}_{6} \mathrm{H}_{4} \mathrm{CO} \mathrm{CH}=\mathrm{CHC}_{6} \mathrm{H}_{4} \mathrm{Br}$ & 369 & 80 & $\begin{array}{c}110-111 \\
(110-111) \\
{[21]}\end{array}$ & --- \\
\hline 27 & $3,5-\mathrm{Cl}_{2}-2-\mathrm{OHC}_{6} \mathrm{H}_{2}$ & $\mathrm{C}_{6} \mathrm{H}_{5}$ & $3,5-\mathrm{Cl}_{2}-2-\mathrm{OH}-\mathrm{C}_{6} \mathrm{H}_{2} \mathrm{COCH}=\mathrm{CHC}_{6} \mathrm{H}_{4}$ & 293 & 83 & $110-111$ & $\begin{array}{c}293\left[\mathrm{M}^{+}\right] \\
295\left[\mathrm{M}^{2+}\right] \\
297\left[\mathrm{M}^{4+}\right] \\
214,201 \\
188,103 \\
91,77\end{array}$ \\
\hline 28 & $3,5-\mathrm{Cl}_{2}-2-\mathrm{OHC}_{6} \mathrm{H}_{2}$ & $3-\mathrm{BrC}_{6} \mathrm{H}_{4}$ & $3,5-\mathrm{Cl}_{2}-2-\mathrm{OHC}_{6} \mathrm{H}_{2} \mathrm{COCH}=\mathrm{CHC}_{6} \mathrm{H}_{4} \mathrm{Br}$ & 372 & 86 & $262-263$ & $\begin{array}{c}372\left[\mathrm{M}^{+}\right], \\
374\left[\mathrm{M}^{2+}\right], \\
376\left[\mathrm{M}^{4+}\right], \\
378\left[\mathrm{M}^{6+}\right], \\
214,201,1 \\
88,180, \\
154,78,77 .\end{array}$ \\
\hline 29 & $3,5-\mathrm{Cl}_{2}-2-\mathrm{OHC}_{6} \mathrm{H}_{2}$ & $4-\mathrm{BrC}_{6} \mathrm{H}_{4}$ & $3,5-\mathrm{Cl}_{2}-2-\mathrm{OHC}_{6} \mathrm{H}_{2} \mathrm{COCH}=\mathrm{CHC}_{6} \mathrm{H}_{4} \mathrm{Br}$ & 372 & 84 & $251-252$ & $\begin{array}{c}372\left[\mathrm{M}^{+}\right], \\
374\left[\mathrm{M}^{2+}\right], \\
376\left[\mathrm{M}^{4+}\right], \\
378\left[\mathrm{M}^{6+}\right], \\
214,201, \\
188,180, \\
154,78,77\end{array}$ \\
\hline 30 & $3,5-\mathrm{Cl}_{2}-2-\mathrm{OHC}_{6} \mathrm{H}_{2}$ & $2-\mathrm{ClC}_{6} \mathrm{H}_{4}$ & $3,5-\mathrm{Cl}_{2}-2-\mathrm{OHC}_{6} \mathrm{H}_{2} \mathrm{CO} \mathrm{CH}=\mathrm{CHC}_{6} \mathrm{H}_{4} \mathrm{Cl}$ & 327 & 86 & $261-262$ & $\begin{array}{c}327\left[\mathrm{M}^{+}\right], \\
329\left[\mathrm{M}^{2+}\right], \\
331\left[\mathrm{M}^{4+}\right] \\
333\left[\mathrm{M}^{6+}\right], \\
214,201, \\
188,180, \\
137,124, \\
111,77,34\end{array}$ \\
\hline
\end{tabular}




\begin{tabular}{|c|c|c|c|c|c|c|c|}
\hline 31 & $3,5-\mathrm{Cl}_{2}-2-\mathrm{OHC}_{6} \mathrm{H}_{2}$ & $3-\mathrm{ClC}_{6} \mathrm{H}_{4}$ & $3,5-\mathrm{Cl}_{2}-2-\mathrm{OHC}_{6} \mathrm{H}_{2} \mathrm{CO} \mathrm{CH}=\mathrm{CHC}_{6} \mathrm{H}_{4} \mathrm{Cl}$ & 327 & 85 & $116-117$ & $\begin{array}{c}327\left[\mathrm{M}^{+}\right] \\
329\left[\mathrm{M}^{2+}\right] \\
331\left[\mathrm{M}^{4+}\right] \\
333\left[\mathrm{M}^{6+}\right] \\
214,201,1 \\
88,180 \\
137,124 \\
111,77,34\end{array}$ \\
\hline 32 & $3,5-\mathrm{Cl}_{2}-2-\mathrm{OHC}_{6} \mathrm{H}_{2}$ & $4-\mathrm{ClC}_{6} \mathrm{H}_{4}$ & $3,5-\mathrm{Cl}_{2}-2-\mathrm{OHC}_{6} \mathrm{H}_{2} \mathrm{COCH}=\mathrm{CHC}_{6} \mathrm{H}_{4} \mathrm{Cl}$ & 327 & 87 & $149-150$ & $\begin{array}{c}327\left[\mathrm{M}^{+}\right] \\
329\left[\mathrm{M}^{+1}\right], \\
331\left[\mathrm{M}^{+}\right] \\
333\left[\mathrm{M}^{6+}\right], \\
214,201,1 \\
88,180, \\
137,124, \\
111,77,34\end{array}$ \\
\hline 33 & $3,5-\mathrm{Cl}_{2}-2-\mathrm{OHC}_{6} \mathrm{H}_{2}$ & 4- $\mathrm{FC}_{6} \mathrm{H}_{4}$ & $3,5-\mathrm{Cl}_{2}-2-\mathrm{OHC}_{6} \mathrm{H}_{2} \mathrm{COCH}=\mathrm{CHC}_{6} \mathrm{H}_{4} \mathrm{~F}$ & 311 & 84 & $110-111$ & $\begin{array}{c}311\left[\mathrm{M}^{+}\right], \\
313\left[\mathrm{M}^{2+}\right] \\
315\left[\mathrm{M}^{4+}\right], \\
214, \\
201,188, \\
121,108, \\
95,77,18 .\end{array}$ \\
\hline 34 & $3,5-\mathrm{Cl}_{2}-2-\mathrm{OHC}_{6} \mathrm{H}_{2}$ & $4-\mathrm{CH}_{3} \mathrm{C}_{6} \mathrm{H}_{4}$ & $3,5-\mathrm{Cl}_{2}-2-\mathrm{OHC}_{6} \mathrm{H}_{2} \mathrm{COCH}=\mathrm{CHC}_{6} \mathrm{H}_{4} \mathrm{CH}_{3}$ & 307 & 85 & $201-202$ & $\begin{array}{c}307\left[\mathrm{M}^{+}\right], \\
309\left[\mathrm{M}^{2+}\right] \\
311\left[\mathrm{M}^{4+}\right], \\
214, \\
201,188, \\
117,104, \\
91,77,15 .\end{array}$ \\
\hline 35 & $3,5-\mathrm{Cl}_{2}-2-\mathrm{OHC}_{6} \mathrm{H}_{2}$ & $3-\mathrm{NO}_{2} \mathrm{C}_{6} \mathrm{H}_{4}$ & $3,5-\mathrm{Cl}_{2}-2-\mathrm{OHC}_{6} \mathrm{H}_{2} \mathrm{COCH}=\mathrm{CHC}_{6} \mathrm{H}_{4}-\mathrm{NO}_{2}$ & 338 & 87 & $232-233$ & $\begin{array}{c}338\left[\mathrm{M}^{+}\right] \\
340\left[\mathrm{M}^{2+}\right] \\
342\left[\mathrm{M}^{4+}\right] \\
214, \\
201,188, \\
148,135, \\
122,7,45 .\end{array}$ \\
\hline 36 & $3,5-\mathrm{Cl}_{2}-2-\mathrm{OHC}_{6} \mathrm{H}_{2}$ & $4-\mathrm{NO}_{2} \mathrm{C}_{6} \mathrm{H}_{4}$ & $3,5-\mathrm{Cl}_{2}-2-\mathrm{OHC}_{6} \mathrm{H}_{2} \mathrm{CO} \mathrm{CH}=\mathrm{CHC}_{6} \mathrm{H}_{4}-\mathrm{NO}_{2}$ & 338 & 88 & $221-222$ & $\begin{array}{c}338\left[\mathrm{M}^{+}\right], \\
340\left[\mathrm{M}^{2+}\right], \\
342\left[\mathrm{M}^{4+}\right], \\
214,201,1 \\
88,148,13 \\
5,122,77, \\
45 .\end{array}$ \\
\hline
\end{tabular}

The Ultra-violet, infrared and NMR spectral data of unknown chalcones, substituted 3,5dichloro-2-hydroxy phenyl ketones were summarized below (27-36). 
(2E)-1-(3,5-Dichloro-2-hydroxyphenyl)-3-phenyl-2-propen-1-one (27): $\mathrm{IR} \quad\left(\mathrm{KBr}, \mathrm{cm}^{-1}\right)$ : $v=1639.17\left(\mathrm{CO}_{\text {s-cis }}\right), 1570.68\left(\mathrm{CO}_{\text {s-trans }}\right), 1044.36(\mathrm{CH}=\mathrm{CH}), 3438.55(\mathrm{OH}) ;{ }^{1} \mathrm{H} \mathrm{NMR}\left(\mathrm{CDCl}_{3}\right.$, ppm): $\delta=7.580(d, 1 \mathrm{H}, \alpha), 8.020(d, 1 \mathrm{H}, \beta), 7.479-7.849(m, 7 \mathrm{H} \mathrm{Ar}-\mathrm{H}), 1.851(s, 1 \mathrm{H}-\mathrm{OH}) ;{ }^{13} \mathrm{C}$ $\operatorname{NMR}\left(\mathrm{CDCl}_{3}, \mathrm{ppm}\right): \delta=121.068\left(\mathrm{C}_{\alpha}\right) 147.619\left(\mathrm{C}_{\beta}\right), 192.607(\mathrm{CO}), 134.110\left(\mathrm{C}_{1}\right), 127.430\left(\mathrm{C}_{2}, \mathrm{C}_{6}\right)$, 127.646 $\left(\mathrm{C}_{3}, \mathrm{C}_{4}, \mathrm{C}_{5}\right), \quad 124.174\left(\mathrm{C}_{1}{ }^{\prime}\right), \quad 157.998\left(\mathrm{C}_{2}{ }^{\prime}\right), \quad 127.430\left(\mathrm{C}_{3}{ }^{\prime}\right), \quad 135.766\left(\mathrm{C}_{4}{ }^{\prime}\right), \quad 128.992\left(\mathrm{C}_{5}{ }^{\prime}\right)$, $129.182\left(\mathrm{C}_{6}^{\prime}\right)$.

(2E)-1-(3,5-Dichloro-2-hydroxyphenyl)-3-(3-bromophenyl)-2-propen-1-one (28): IR ( $\mathrm{KBr}$, $\left.\mathrm{cm}^{-1}\right): \quad v=1648.64\left(\mathrm{CO}_{\text {s-cis }}\right), \quad 1583.65\left(\mathrm{CO}_{\text {s-trans }}\right), \quad 1026.02(\mathrm{CH}=\mathrm{CH}), \quad 3423.39(\mathrm{OH}) ;{ }^{1} \mathrm{H} \quad \mathrm{NMR}$ $\left(\mathrm{CDCl}_{3}, \mathrm{ppm}\right): \delta=7.554(d, 1 \mathrm{H}, \alpha), 7.916(d, 1 \mathrm{H}, \beta), 7.281-7.931(\mathrm{~m}, 6 \mathrm{H} \mathrm{Ar}-\mathrm{H}), 3.535(\mathrm{~s}, 1 \mathrm{H}-$ $\mathrm{OH}) ;{ }^{13} \mathrm{C} \mathrm{NMR}\left(\mathrm{CDCl}_{3}, \mathrm{ppm}\right): \delta=131.155\left(\mathrm{C}_{\alpha}\right) \quad 141.319\left(\mathrm{C}_{\beta}\right), 192.541(\mathrm{CO}), 135.995\left(\mathrm{C}_{1}\right)$, 131.155( $\left.\mathrm{C}_{2}\right), 131.155\left(\mathrm{C}_{3}\right), 131.155\left(\mathrm{C}_{4}\right), 131.155\left(\mathrm{C}_{5}\right), 131.155\left(\mathrm{C}_{6}\right), 131.155\left(\mathrm{C}_{1}{ }^{\prime}\right), 150.030\left(\mathrm{C}_{2}{ }^{\prime}\right)$, 131.155( $\left(\mathrm{C}_{3}{ }^{\prime}\right), 135.995\left(\mathrm{C}_{4}{ }^{\prime}\right), 134.301\left(\mathrm{C}_{5}{ }^{\prime}\right), 134.301\left(\mathrm{C}_{6}{ }^{\prime}\right)$.

(2E)-1-(3, 5-Dichloro-2-hydroxyphenyl)-3-(4-bromophenyl)-2-propen-1-one (29): IR ( $\mathrm{KBr}$, $\left.\mathrm{cm}^{-1}\right): \quad v=1643.77\left(\mathrm{CO}_{\text {s-cis }}\right), \quad 1574.29\left(\mathrm{CO}_{\text {s-trans }}\right), \quad 1045.73(\mathrm{CH}=\mathrm{CH}), \quad 3459.64(\mathrm{OH}) ;{ }^{1} \mathrm{H} \quad \mathrm{NMR}$ $\left(\mathrm{CDCl}_{3}, \mathrm{ppm}\right): \delta=7.555(d, 1 \mathrm{H}, \alpha), 7.934(d, 1 \mathrm{H}, \beta), 7.285-7.857(m, 6 \mathrm{H} \mathrm{Ar}-\mathrm{H}), 4.707(s, 1 \mathrm{H}-$ $\mathrm{OH}) ;{ }^{13} \mathrm{C} \mathrm{NMR}\left(\mathrm{CDCl}_{3}, \mathrm{ppm}\right): \delta=120.957\left(\mathrm{C}_{\alpha}\right) \quad 146.090\left(\mathrm{C}_{\beta}\right), 192.351(\mathrm{CO}), 135.912\left(\mathrm{C}_{1}\right)$, 127.627 $\left(\mathrm{C}_{2}, \mathrm{C}_{6}\right), \quad 132.155\left(\mathrm{C}_{3}, \mathrm{C}_{5}\right), \quad 123.363\left(\mathrm{C}_{4}\right), \quad 126.086\left(\mathrm{C}_{1}{ }^{\prime}\right), \quad 158.017\left(\mathrm{C}_{2}{ }^{\prime}\right), \quad 127.365\left(\mathrm{C}_{3}{ }^{\prime}\right)$, 135.912( $\left(\mathrm{C}_{4}{ }^{\prime}\right), 127.627\left(\mathrm{C}_{5}{ }^{\prime}\right), 130.253\left(\mathrm{C}_{6}{ }^{\prime}\right)$.

(2E)-1-(3,5-Dichloro-2-hydroxyphenyl)-3-(2-chlorophenyl)-2-propen-1-one (30): IR ( $\mathrm{KBr}$, $\left.\mathrm{cm}^{-1}\right): \quad v=1644.06\left(\mathrm{CO}_{\text {s-cis }}\right), \quad 1579.14\left(\mathrm{CO}_{\text {s-trans }}\right), \quad 1026.37(\mathrm{CH}=\mathrm{CH}), \quad 3429.16(\mathrm{OH}) ;{ }^{1} \mathrm{H} \quad \mathrm{NMR}$ $\left(\mathrm{CDCl}_{3}, \mathrm{ppm}\right): \delta=7.566(d, 1 \mathrm{H}, \alpha), 8.413(d, 1 \mathrm{H}, \beta), 7.285-7.688(\mathrm{~m}, 6 \mathrm{H} \mathrm{Ar}-\mathrm{H}), 3.046(s, 1 \mathrm{H}-$ $\mathrm{OH}) ;{ }^{13} \mathrm{C} \mathrm{NMR}\left(\mathrm{CDCl}_{3}, \mathrm{ppm}\right): \delta=129.703\left(\mathrm{C}_{\alpha}\right) \quad 146.642\left(\mathrm{C}_{\beta}\right), 193.603(\mathrm{CO}), 135.995\left(\mathrm{C}_{1}\right)$, 131.155( $\left.\mathrm{C}_{2}\right), 131.155\left(\mathrm{C}_{3}\right), 131.155\left(\mathrm{C}_{4}\right), 131.155\left(\mathrm{C}_{5}\right), 131.155\left(\mathrm{C}_{6}\right), 131.155\left(\mathrm{C}_{1}{ }^{\prime}\right), 150.030\left(\mathrm{C}_{2}{ }^{\prime}\right)$, 131.155( $\left(\mathrm{C}_{3}{ }^{\prime}\right), 135.995\left(\mathrm{C}_{4}{ }^{\prime}\right), 134.301\left(\mathrm{C}_{5}{ }^{\prime}\right), 134.301\left(\mathrm{C}_{6}{ }^{\prime}\right)$.

(2E)-1-(3,5-Dichloro-2-hydroxyphenyl)-3-(3-chlorophenyl)-2-propen-1-one (31): IR ( $\mathrm{KBr}$, $\left.\mathrm{cm}^{-1}\right): \quad v=1645.69\left(\mathrm{CO}_{\text {s-cis }}\right), \quad 1585.31\left(\mathrm{CO}_{\text {s-trans }}\right), \quad 1034.67(\mathrm{CH}=\mathrm{CH}), \quad 3455.43(\mathrm{OH}) ;{ }^{1} \mathrm{H} \quad \mathrm{NMR}$ $\left(\mathrm{CDCl}_{3}, \mathrm{ppm}\right): \delta=7.561(d, 1 \mathrm{H}, \alpha), 7.927(d, 1 \mathrm{H}, \beta), 7.369-7.701(\mathrm{~m}, 6 \mathrm{H} \mathrm{Ar}-\mathrm{H}), 5.556(s, 1 \mathrm{H}-$ $\mathrm{OH}) ;{ }^{13} \mathrm{C} \mathrm{NMR}\left(\mathrm{CDCl}_{3}, \mathrm{ppm}\right): \delta=120.323\left(\mathrm{C}_{\alpha}\right) 145.742\left(\mathrm{C}_{\beta}\right), 192.322(\mathrm{CO}), 136.004\left(\mathrm{C}_{1}\right)$, $127.139\left(\mathrm{C}_{2}\right), 134.995\left(\mathrm{C}_{3}\right), 128.354\left(\mathrm{C}_{4}\right), 130.415\left(\mathrm{C}_{5}\right), 124.307\left(\mathrm{C}_{6}\right), 125.139\left(\mathrm{C}_{1}{ }^{\prime}\right), 158.018\left(\mathrm{C}_{2}{ }^{\prime}\right)$, $126.182\left(\mathrm{C}_{3}{ }^{\prime}\right), 136.004\left(\mathrm{C}_{4}{ }^{\prime}\right), 129.152\left(\mathrm{C}_{5}{ }^{\prime}\right), 130.279\left(\mathrm{C}_{6}{ }^{\prime}\right)$.

(2E)-1-(3,5-Dichloro-2-hydroxyphenyl)-3-(4-chlorophenyl)-2-propen-1-one (32): IR ( $\mathrm{KBr}$, $\left.\mathrm{cm}^{-1}\right): \quad v=1638.60\left(\mathrm{CO}_{\text {s-cis }}\right), \quad 1581.57\left(\mathrm{CO}_{\text {s-trans }}\right), \quad 1015.80(\mathrm{CH}=\mathrm{CH}), \quad 3450.09(\mathrm{OH}) ;{ }^{1} \mathrm{H} \quad \mathrm{NMR}$ $\left(\mathrm{CDCl}_{3}, \mathrm{ppm}\right): \delta=7.536(d, 1 \mathrm{H}, \alpha), 7.948(d, 1 \mathrm{H}, \beta), 7.284-7.964(m, 6 \mathrm{H} \mathrm{Ar}-\mathrm{H}), 4.696(s, 1 \mathrm{H}-$ $\mathrm{OH}) ;{ }^{13} \mathrm{C} \mathrm{NMR}\left(\mathrm{CDCl}_{3}, \mathrm{ppm}\right): \delta=120.962\left(\mathrm{C}_{\alpha}\right) \quad 146.007\left(\mathrm{C}_{\beta}\right), 192.338(\mathrm{CO}), 132.566\left(\mathrm{C}_{1}\right)$, 127.352 $\left(\mathrm{C}_{2}, \mathrm{C}_{6}\right), \quad 129.191\left(\mathrm{C}_{3}\right), \quad 134.927\left(\mathrm{C}_{4}\right), \quad 129.504\left(\mathrm{C}_{5}\right), \quad 124.245\left(\mathrm{C}_{1}{ }^{\prime}\right), \quad 158.013\left(\mathrm{C}_{2}{ }^{\prime}\right)$, $125.124\left(\mathrm{C}_{3}^{\prime}\right), 137.642\left(\mathrm{C}_{4}{ }^{\prime}\right), 127.062\left(\mathrm{C}_{5}{ }^{\prime}\right), 129.504\left(\mathrm{C}_{6}{ }^{\prime}\right)$.

(2E)-1-(3,5-Dichloro-2-hydroxyphenyl)-3-(4-fluorophenyl)-2-propen-1-one (33): IR ( $\mathrm{KBr}$, $\left.\mathrm{cm}^{-1}\right): \quad v=1645.58\left(\mathrm{CO}_{\text {s-cis }}\right), \quad 1582.36\left(\mathrm{CO}_{\text {s-trans }}\right), \quad 1020.64(\mathrm{CH}=\mathrm{CH}), \quad 3431.29(\mathrm{OH}) ;{ }^{1} \mathrm{H} \quad \mathrm{NMR}$ $\left(\mathrm{CDCl}_{3}, \mathrm{ppm}\right): \delta=7.503(d, 1 \mathrm{H}, \alpha), 7.981(d, 1 \mathrm{H}, \beta), 7.162-7.997(m, 6 \mathrm{H} \mathrm{Ar}-\mathrm{H}), 3.921(s, 1 \mathrm{H}-$ $\mathrm{OH}) ;{ }^{13} \mathrm{C} \mathrm{NMR}\left(\mathrm{CDCl}_{3}, \mathrm{ppm}\right): \delta=127.365\left(\mathrm{C}_{\alpha}\right) \quad 146.400\left(\mathrm{C}_{\beta}\right), 192.417(\mathrm{CO}), 130.999\left(\mathrm{C}_{1}\right)$, 127.365 $\left(\mathrm{C}_{2}, \mathrm{C}_{6}\right), \quad 116.636\left(\mathrm{C}_{3}, \mathrm{C}_{5}\right), \quad 164.307\left(\mathrm{C}_{4}\right), \quad 127.365\left(\mathrm{C}_{1}{ }^{\prime}\right), \quad 164.307\left(\mathrm{C}_{2}{ }^{\prime}\right), \quad 127.365\left(\mathrm{C}_{3}{ }^{\prime}\right)$, 135.995( $\left.\mathrm{C}_{4}{ }^{\prime}\right), 127.365\left(\mathrm{C}_{5}{ }^{\prime}\right), 130.999\left(\mathrm{C}_{6}{ }^{\prime}\right)$. 
(2E)-1-(3,5-Dichloro-2-hydroxyphenyl)-3-(4-methylphenyl)-2-propen-1-one (34): IR ( $\mathrm{KBr}$, $\left.\mathrm{cm}^{-1}\right): \quad v=1636.89\left(\mathrm{CO}_{\text {s-cis }}\right), \quad 1590.49\left(\mathrm{CO}_{\text {s-trans }}\right), \quad 1054.97(\mathrm{CH}=\mathrm{CH}), \quad 3435.69(\mathrm{OH}) ;{ }^{1} \mathrm{H} \quad \mathrm{NMR}$ $\left(\mathrm{CDCl}_{3}, \mathrm{ppm}\right): \delta=7.386(d, 1 \mathrm{H}, \alpha), 7.641(d, 1 \mathrm{H}, \beta), 6.951-7.657(m, 6 \mathrm{H} \mathrm{Ar}-\mathrm{H}), 3.873(s, 1 \mathrm{H}-$ $\mathrm{OH}), 2.402\left(\mathrm{~s}, 3 \mathrm{H}-\mathrm{CH}_{3}\right) ;{ }^{13} \mathrm{C} \mathrm{NMR}\left(\mathrm{CDCl}_{3}, \mathrm{ppm}\right): \delta=118.947\left(\mathrm{C}_{\alpha}\right) 143.564\left(\mathrm{C}_{\beta}\right), 192.645(\mathrm{CO})$, 132.411 $\left(\mathrm{C}_{1}\right), 126.010\left(\mathrm{C}_{2}, \mathrm{C}_{6}\right), 129.634\left(\mathrm{C}_{3} \mathrm{C}_{5}\right), 140.771\left(\mathrm{C}_{4}\right), 126.010\left(\mathrm{C}_{1}{ }^{\prime}, \mathrm{C}_{3}{ }^{\prime}\right), 153.654\left(\mathrm{C}_{2}{ }^{\prime}\right)$, 132.411( $\left.\mathrm{C}_{4}^{\prime}\right), 128.456\left(\mathrm{C}_{5}^{\prime}\right), 129.901\left(\mathrm{C}_{6}^{\prime}\right), 21.501\left(\mathrm{CH}_{3}\right)$.

(2E)-1-(3,5-Dichloro-2-hydroxyphenyl)-3-(3-nitrolphenyl)-2-propen-1-one (35): $\mathrm{IR}$ ( $\mathrm{KBr}$, $\left.\mathrm{cm}^{-1}\right): \quad v=1650.15\left(\mathrm{CO}_{\text {s-cis }}\right), \quad 1585.00\left(\mathrm{CO}_{\text {s-trans }}\right), \quad 1045.68(\mathrm{CH}=\mathrm{CH}), \quad 3452.09(\mathrm{OH}) ;{ }^{1} \mathrm{H} \quad \mathrm{NMR}$ $\left(\mathrm{CDCl}_{3}, \mathrm{ppm}\right): \delta=7.865(d, 1 \mathrm{H}, \alpha), 8.027(d, 1 \mathrm{H}, \beta), 7.600-8.584(m, 6 \mathrm{H} \mathrm{Ar}-\mathrm{H}), 2.682(s, 1 \mathrm{H}-$ $\mathrm{OH}) ;{ }^{13} \mathrm{C} \mathrm{NMR}\left(\mathrm{CDCl}_{3}, \mathrm{ppm}\right): \delta=122.928\left(\mathrm{C}_{\alpha}\right) \quad 140.835\left(\mathrm{C}_{\beta}\right), 192.873(\mathrm{CO}), 140.835\left(\mathrm{C}_{1}\right)$, $122.928\left(\mathrm{C}_{2}\right), 140.835\left(\mathrm{C}_{3}\right), 122.928\left(\mathrm{C}_{4}\right), 122.928\left(\mathrm{C}_{5}\right), 140.835\left(\mathrm{C}_{6}\right), 122.928\left(\mathrm{C}_{1}{ }^{\prime}\right), 169.147\left(\mathrm{C}_{2}{ }^{\prime}\right)$, $122.928\left(\mathrm{C}_{3}{ }^{\prime}\right), 140.835\left(\mathrm{C}_{4}{ }^{\prime}\right), 122.928\left(\mathrm{C}_{5}{ }^{\prime}\right), 122.958\left(\mathrm{C}_{6}{ }^{\prime}\right)$.

(2E)-1-(3,5-Dichloro-2-hydroxyphenyl)-3-(4-nitrolphenyl)-2-propen-1-one (36): $\mathrm{IR}$ ( $\mathrm{KBr}$, $\left.\mathrm{cm}^{-1}\right): \quad v=1647.51\left(\mathrm{CO}_{\text {s-cis }}\right), \quad 1586.57\left(\mathrm{CO}_{\text {s-trans }}\right), \quad 1041.17(\mathrm{CH}=\mathrm{CH}), \quad 3435.07(\mathrm{OH}) ;{ }^{1} \mathrm{H} \quad \mathrm{NMR}$ $\left(\mathrm{CDCl}_{3}, \mathrm{ppm}\right): \delta=7.864(d, 1 \mathrm{H}, \alpha), 8.013(d, 1 \mathrm{H}, \beta), 7.652-7.879(m, 6 \mathrm{H} \mathrm{Ar}-\mathrm{H}), 3.097(s, 1 \mathrm{H}-$ $\mathrm{OH}) ;{ }^{13} \mathrm{C} \mathrm{NMR}\left(\mathrm{CDCl}_{3}, \mathrm{ppm}\right): \delta=124.374\left(\mathrm{C}_{\alpha}\right) \quad 152.692\left(\mathrm{C}_{\beta}\right), 193.435(\mathrm{CO}), 134.301\left(\mathrm{C}_{1}\right)$, $129.446\left(\mathrm{C}_{2}, \mathrm{C}_{6}\right), \quad 124.374\left(\mathrm{C}_{3}, \mathrm{C}_{5}\right), \quad 152.692\left(\mathrm{C}_{4}\right), 114.459\left(\mathrm{C}_{1}{ }^{\prime}\right), 159.226\left(\mathrm{C}_{2}{ }^{\prime}\right), 124.374\left(\mathrm{C}_{3}{ }^{\prime}\right)$, $134.301\left(\mathrm{C}_{4}^{\prime}\right), 129.446\left(\mathrm{C}_{5}^{\prime}, \mathrm{C}_{6}^{\prime},\right)$.

In the present investigation UV absorption maxima form UV spectra, the spectral linearity of chalcones has been studied by evaluating the effect of substituents. The assigned group frequencies of all chalcones like carbonyl stretches $v \mathrm{CO}$, the deformation modes of vinyl part $\mathrm{CH}$ out of plane, in-plane, $\mathrm{CH}=\mathrm{CH}$ and $>\mathrm{C}=\mathrm{C}<$ out of planes $\left(\mathrm{cm}^{-1}\right)$, the vinyl hydrogen from IR spectra and chemical shifts $\delta(\mathrm{ppm})$, of $\mathrm{H}_{\alpha}, \mathrm{H}_{\beta}, \mathrm{C}_{\alpha}, \mathrm{C}_{\beta}$ and $\mathrm{CO}$ from ${ }^{1} \mathrm{H}$ and ${ }^{13} \mathrm{C} \mathrm{NMR}$ spectra have been correlated with various substituent constants.

\section{1. Ultra violet spectral study}

The UV spectra of all synthesized chalcones were recorded in SHIMADZU-1650 SPECTROMETER $\left(\lambda_{\max } \mathrm{nm}\right)$ in spectral grade methanol. The measured absorption maxima $\left(\lambda_{\max } \mathrm{nm}\right)$ of these chalcones are presented in Table 2 . These values are correlated with Hammett substituent constants and $\mathrm{F}$ and $\mathrm{R}$ parameters using single and multi-linear regression analysis. Hammett correlation involving the group frequencies and absorption maxima, the form of the Hammett equation employed is

$$
\lambda=\rho \sigma+\lambda_{0}
$$

where $\lambda_{0}$ is the frequency for the parent member of the series.

The results of statistical analysis of these values with Hammett substituent constants are presented in Table 3. From Table 3, Hammett substituent constants $\sigma, \sigma^{+}, \sigma_{\mathrm{I}}, \sigma_{\mathrm{R}}, \mathrm{F}$ and $\mathrm{R}$ values gave poor correlations with $\lambda \max$. All constants gave negative $\rho$ values. This is due to 
Table 2. The Uv $\lambda_{\max }(\mathrm{nm})$, IR $v\left(\mathrm{~cm}^{-1}\right)$, and NMR $\delta(\mathrm{ppm})$ frequencies of substituted styryl 3,5-dichloro-2-hydroxyphenyl ketones (entries 27-36).

\begin{tabular}{|c|c|c|c|c|c|c|c|c|c|c|c|c|c|}
\hline$\underset{\Xi}{\stackrel{\Xi}{\Xi}}$ & 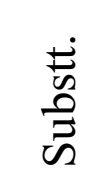 & ڤ゙્ & $\bigcup_{0}^{\substack{u \\
i}}$ & 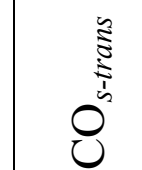 & $\stackrel{3}{J}$ & $\underbrace{8}_{\supset}$ & $\underbrace{\infty}_{\mathbb{I}}$ & & $\frac{\partial}{\infty}$ & $\frac{n}{\infty}$ & $\bigcup_{\infty}^{0}$ & $U_{0}^{8}$ & $\bigcup_{0}^{\infty}$ \\
\hline 27 & $\mathrm{H}$ & 318.0 & 1639.17 & 1570.68 & 1169.73 & 770.42 & 1044.36 & 590.98 & 7.580 & 8.020 & 192.60 & 121.06 & 147.61 \\
\hline 28 & $3-\mathrm{Br}$ & 275.2 & 1648.64 & 1583.65 & 1144.22 & 749.71 & 1026.02 & 573.75 & 7.554 & 7.916 & 192.54 & 131.15 & 141.31 \\
\hline 29 & $4-\mathrm{Br}$ & 322.0 & 1643.77 & 1574.29 & 1167.94 & 745.78 & 1045.73 & 601.71 & 7.555 & 7.934 & 192.35 & 120.95 & 146.09 \\
\hline 30 & $2-\mathrm{Cl}$ & 285.9 & 1644.06 & 1579.14 & 1139.97 & 749.09 & 1026.37 & 581.89 & 7.566 & 8.413 & 193.60 & 129.70 & 146.64 \\
\hline 31 & $3-\mathrm{Cl}$ & 270.8 & 1645.69 & 1585.31 & 1167.76 & 786.84 & 1034.67 & 602.94 & 7.561 & 7.927 & 192.32 & 120.32 & 145.74 \\
\hline 32 & $4-\mathrm{Cl}$ & 329.4 & 1638.60 & 1581.57 & 1168.64 & 749.84 & 1015.8 & 534.47 & 7.536 & 7.948 & 192.33 & 120.96 & 146.00 \\
\hline 33 & $4-F$ & 327.0 & 1645.58 & 1582.36 & 1157.51 & 755.68 & 1020.64 & 542.47 & 7.503 & 7.981 & 192.41 & 127.36 & 146.40 \\
\hline 34 & 4- $\mathrm{CH}_{3}$ & 334.2 & 1636.89 & 1590.49 & 1172.62 & 738.87 & 1054.97 & 571.86 & 7.386 & 7.641 & 192.64 & 118.94 & 143.56 \\
\hline 35 & $3-\mathrm{NO}_{2}$ & 270.6 & 1650.15 & 1585.00 & 1149.89 & 751.69 & 1045.68 & 546.72 & 7.865 & 8.027 & 192.87 & 122.92 & 140.83 \\
\hline 36 & $4-\mathrm{NO}_{2}$ & 315.6 & 1647.51 & 1586.57 & 1164.22 & 745.76 & 1041.17 & 558.90 & 7.864 & 8.013 & 193.43 & 124.37 & 152.69 \\
\hline
\end{tabular}


Table 3. Results of statistical analysis of substituted styryl 3,5-dichloro-2-hydroxyphenyl ketones (entries 27-36).

\begin{tabular}{|c|c|c|c|c|c|c|c|}
\hline 己̇ & 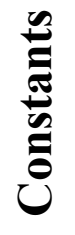 & $\mathbf{r}$ & $\mathbf{I}$ & $\boldsymbol{\rho}$ & $\mathbf{S}$ & $\mathbf{n}$ & Correlated derivatives \\
\hline \multirow{6}{*}{$\underbrace{3}_{5}$} & $\sigma$ & 0.854 & 318.12 & -47.119 & 23.12 & 10 & $\mathrm{H}, 3-\mathrm{Br}, 4-\mathrm{Br}, 2-\mathrm{Cl}, 3-\mathrm{Cl}, 4-\mathrm{Cl}, 4-\mathrm{F}, 4-\mathrm{CH}_{3}, 3-\mathrm{NO}_{2}, 4-\mathrm{NO}_{2}$ \\
\hline & $\sigma^{+}$ & 0.862 & 318.38 & -46.439 & 21.52 & 10 & $\mathrm{H}, 3-\mathrm{Br}, 4-\mathrm{Br}, 2-\mathrm{Cl}, 3-\mathrm{Cl}, 4-\mathrm{Cl}, 4-\mathrm{F}, 4-\mathrm{CH}_{3}, 3-\mathrm{NO}_{2}, 4-\mathrm{NO}_{2}$ \\
\hline & $\sigma_{\mathrm{I}}$ & 0.842 & 323.76 & -45.759 & 25.05 & 10 & $\mathrm{H}, 3-\mathrm{Br}, 4-\mathrm{Br}, 2-\mathrm{Cl}, 3-\mathrm{Cl}, 4-\mathrm{Cl}, 4-\mathrm{F}, 4-\mathrm{CH}_{3}, 3-\mathrm{NO}_{2}, 4-\mathrm{NO}_{2}$ \\
\hline & $\sigma_{\mathrm{R}}$ & 0.620 & 301.43 & -28.622 & 27.04 & 10 & $\mathrm{H}, 3-\mathrm{Br}, 4-\mathrm{Br}, 2-\mathrm{Cl}, 3-\mathrm{Cl}, 4-\mathrm{Cl}, 4-\mathrm{F}, 4-\mathrm{CH}_{3}, 3-\mathrm{NO}_{2}, 4-\mathrm{NO}_{2}$ \\
\hline & $\mathrm{F}$ & 0.727 & 317.20 & -29.303 & 26.53 & 10 & $\mathrm{H}, 3-\mathrm{Br}, 4-\mathrm{Br}, 2-\mathrm{Cl}, 3-\mathrm{Cl}, 4-\mathrm{Cl}, 4-\mathrm{F}, 4-\mathrm{CH}_{3}, 3-\mathrm{NO}_{2}, 4-\mathrm{NO}_{2}$ \\
\hline & $\mathrm{R}$ & 0.725 & 299.91 & -31.580 & 26.71 & 10 & $\mathrm{H}, 3-\mathrm{Br}, 4-\mathrm{Br}, 2-\mathrm{Cl}, 3-\mathrm{Cl}, 4-\mathrm{Cl}, 4-\mathrm{F}, 4-\mathrm{CH}_{3}, 3-\mathrm{NO}_{2}, 4-\mathrm{NO}_{2}$ \\
\hline \multirow{6}{*}{ 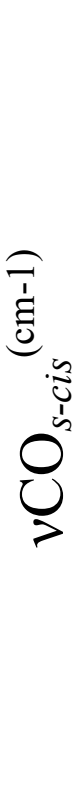 } & $\sigma$ & 0.980 & 1640.66 & 11.833 & 2.84 & 10 & $\mathrm{H}, 3-\mathrm{Br}, 4-\mathrm{Br}, 2-\mathrm{Cl}, 3-\mathrm{Cl}, 4-\mathrm{Cl}, 4-\mathrm{F}, 4-\mathrm{CH}_{3}, 3-\mathrm{NO}_{2}, 4-\mathrm{NO}_{2}$ \\
\hline & $\sigma^{+}$ & 0.979 & 1641.07 & 10.085 & 2.89 & 10 & $\mathrm{H}, 3-\mathrm{Br}, 4-\mathrm{Br}, 2-\mathrm{Cl}, 3-\mathrm{Cl}, 4-\mathrm{Cl}, 4-\mathrm{F}, 4-\mathrm{CH}_{3}, 3-\mathrm{NO}_{2}, 4-\mathrm{NO}_{2}$ \\
\hline & $\sigma_{\mathrm{I}}$ & 0.900 & 1637.80 & 15.019 & 2.81 & 9 & $\mathrm{H}, 3-\mathrm{Br}, 4-\mathrm{Br}, 2-\mathrm{Cl}, 3-\mathrm{Cl}, 4-\mathrm{F}, 4-\mathrm{CH}_{3}, 3-\mathrm{NO}_{2}, 4-\mathrm{NO}_{2}$ \\
\hline & $\sigma_{\mathrm{R}}$ & 0.819 & 1644.56 & 4.658 & 4.65 & 10 & $\mathrm{H}, 3-\mathrm{Br}, 4-\mathrm{Br}, 2-\mathrm{Cl}, 3-\mathrm{Cl}, 4-\mathrm{Cl}, 4-\mathrm{F}, 4-\mathrm{CH}_{3}, 3-\mathrm{NO}_{2}, 4-\mathrm{NO}_{2}$ \\
\hline & $\mathrm{F}$ & 0.710 & 1638.08 & 14.073 & 2.96 & 10 & $\mathrm{H}, 3-\mathrm{Br}, 4-\mathrm{Br}, 2-\mathrm{Cl}, 3-\mathrm{Cl}, 4-\mathrm{Cl}, 4-\mathrm{F}, 4-\mathrm{CH}_{3}, 3-\mathrm{NO}_{2}, 4-\mathrm{NO}_{2}$ \\
\hline & $\mathrm{R}$ & 0.715 & 1644.52 & 3.281 & 4.68 & 10 & $\mathrm{H}, 3-\mathrm{Br}, 4-\mathrm{Br}, 2-\mathrm{Cl}, 3-\mathrm{Cl}, 4-\mathrm{Cl}, 4-\mathrm{F}, 4-\mathrm{CH}_{3}, 3-\mathrm{NO}_{2}, 4-\mathrm{NO}_{2}$ \\
\hline
\end{tabular}




\begin{tabular}{|c|c|c|c|c|c|c|c|}
\hline \multirow{6}{*}{ 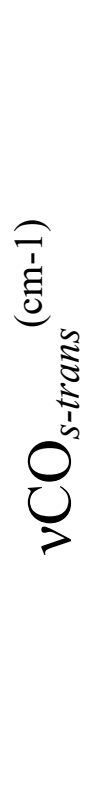 } & $\sigma$ & 0.725 & 1580.51 & 4.939 & 6.03 & 10 & $\mathrm{H}, 3-\mathrm{Br}, 4-\mathrm{Br}, 2-\mathrm{Cl}, 3-\mathrm{Cl}, 4-\mathrm{Cl}, 4-\mathrm{F}, 4-\mathrm{CH}_{3}, 3-\mathrm{NO}_{2}, 4-\mathrm{NO}_{2}$ \\
\hline & $\sigma^{+}$ & 0.705 & 1581.65 & 0.854 & 6.23 & 10 & $\mathrm{H}, 3-\mathrm{Br}, 4-\mathrm{Br}, 2-\mathrm{Cl}, 3-\mathrm{Cl}, 4-\mathrm{Cl}, 4-\mathrm{F}, 4-\mathrm{CH}_{3}, 3-\mathrm{NO}_{2}, 4-\mathrm{NO}_{2}$ \\
\hline & $\sigma_{\mathrm{I}}$ & 0.718 & 1580.07 & 4.428 & 6.13 & 10 & $\mathrm{H}, 3-\mathrm{Br}, 4-\mathrm{Br}, 2-\mathrm{Cl}, 3-\mathrm{Cl}, 4-\mathrm{Cl}, 4-\mathrm{F}, 4-\mathrm{CH}_{3}, 3-\mathrm{NO}_{2}, 4-\mathrm{NO}_{2}$ \\
\hline & $\sigma_{\mathrm{R}}$ & 0.710 & 1582.29 & 3.234 & 6.20 & 10 & $\mathrm{H}, 3-\mathrm{Br}, 4-\mathrm{Br}, 2-\mathrm{Cl}, 3-\mathrm{Cl}, 4-\mathrm{Cl}, 4-\mathrm{F}, 4-\mathrm{CH}_{3}, 3-\mathrm{NO}_{2}, 4-\mathrm{NO}_{2}$ \\
\hline & $\mathrm{F}$ & 0.722 & 1579.66 & 5.318 & 6.08 & 10 & $\mathrm{H}, 3-\mathrm{Br}, 4-\mathrm{Br}, 2-\mathrm{Cl}, 3-\mathrm{Cl}, 4-\mathrm{Cl}, 4-\mathrm{F}, 4-\mathrm{CH}_{3}, 3-\mathrm{NO}_{2}, 4-\mathrm{NO}_{2}$ \\
\hline & $\mathrm{R}$ & 0.804 & 1582.11 & 1.334 & 6.23 & 10 & $\mathrm{H}, 3-\mathrm{Br}, 4-\mathrm{Br}, 2-\mathrm{Cl}, 3-\mathrm{Cl}, 4-\mathrm{Cl}, 4-\mathrm{F}, 4-\mathrm{CH}_{3}, 3-\mathrm{NO}_{2}, 4-\mathrm{NO}_{2}$ \\
\hline \multirow{6}{*}{ 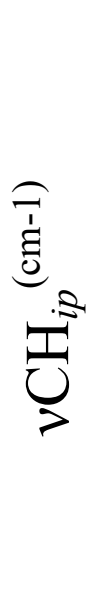 } & $\sigma$ & 0.833 & 1163.88 & -12.881 & 11.67 & 10 & $\mathrm{H}, 3-\mathrm{Br}, 4-\mathrm{Br}, 2-\mathrm{Cl}, 3-\mathrm{Cl}, 4-\mathrm{Cl}, 4-\mathrm{F}, 4-\mathrm{CH}_{3}, 3-\mathrm{NO}_{2}, 4-\mathrm{NO}_{2}$ \\
\hline & $\sigma^{+}$ & 0.845 & 1164.59 & -14.944 & 11.06 & 10 & $\mathrm{H}, 3-\mathrm{Br}, 4-\mathrm{Br}, 2-\mathrm{Cl}, 3-\mathrm{Cl}, 4-\mathrm{Cl}, 4-\mathrm{F}, 4-\mathrm{CH}_{3}, 3-\mathrm{NO}_{2}, 4-\mathrm{NO}_{2}$ \\
\hline & $\sigma_{\mathrm{I}}$ & 0.748 & 1170.02 & -23.659 & 10.82 & 10 & $\mathrm{H}, 3-\mathrm{Br}, 4-\mathrm{Br}, 2-\mathrm{Cl}, 3-\mathrm{Cl}, 4-\mathrm{Cl}, 4-\mathrm{F}, 4-\mathrm{CH}_{3}, 3-\mathrm{NO}_{2}, 4-\mathrm{NO}_{2}$ \\
\hline & $\sigma_{\mathrm{R}}$ & 0.712 & 1161.18 & 7.773 & 12.29 & 10 & $\mathrm{H}, 3-\mathrm{Br}, 4-\mathrm{Br}, 2-\mathrm{Cl}, 3-\mathrm{Cl}, 4-\mathrm{Cl}, 4-\mathrm{F}, 4-\mathrm{CH}_{3}, 3-\mathrm{NO}_{2}, 4-\mathrm{NO}_{2}$ \\
\hline & $\mathrm{F}$ & 0.844 & 1169.09 & -21.010 & 11.08 & 10 & $\mathrm{H}, 3-\mathrm{Br}, 4-\mathrm{Br}, 2-\mathrm{Cl}, 3-\mathrm{Cl}, 4-\mathrm{Cl}, 4-\mathrm{F}, 4-\mathrm{CH}_{3}, 3-\mathrm{NO}_{2}, 4-\mathrm{NO}_{2}$ \\
\hline & $\mathrm{R}$ & 0.809 & 1161.05 & 5.141 & 12.33 & 10 & $\mathrm{H}, 3-\mathrm{Br}, 4-\mathrm{Br}, 2-\mathrm{Cl}, 3-\mathrm{Cl}, 4-\mathrm{Cl}, 4-\mathrm{F}, 4-\mathrm{CH}_{3}, 3-\mathrm{NO}_{2}, 4-\mathrm{NO}_{2}$ \\
\hline \multirow{6}{*}{ 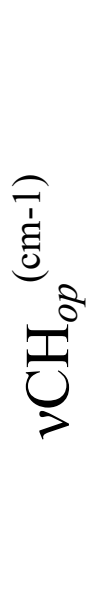 } & $\sigma$ & 0.800 & 754.32 & 0.100 & 14.91 & 10 & $\mathrm{H}, 3-\mathrm{Br}, 4-\mathrm{Br}, 2-\mathrm{Cl}, 3-\mathrm{Cl}, 4-\mathrm{Cl}, 4-\mathrm{F}, 4-\mathrm{CH}_{3}, 3-\mathrm{NO}_{2}, 4-\mathrm{NO}_{2}$ \\
\hline & $\sigma^{+}$ & 0.701 & 754.18 & 0.635 & 14.91 & 10 & $\mathrm{H}, 3-\mathrm{Br}, 4-\mathrm{Br}, 2-\mathrm{Cl}, 3-\mathrm{Cl}, 4-\mathrm{Cl}, 4-\mathrm{F}, 4-\mathrm{CH}_{3}, 3-\mathrm{NO}_{2}, 4-\mathrm{NO}_{2}$ \\
\hline & $\sigma_{\mathrm{I}}$ & 0.706 & 755.84 & -3.587 & 14.88 & 10 & $\mathrm{H}, 3-\mathrm{Br}, 4-\mathrm{Br}, 2-\mathrm{Cl}, 3-\mathrm{Cl}, 4-\mathrm{Cl}, 4-\mathrm{F}, 4-\mathrm{CH}_{3}, 3-\mathrm{NO}_{2}, 4-\mathrm{NO}_{2}$ \\
\hline & $\sigma_{\mathrm{R}}$ & 0.709 & 753.47 & -7.414 & 14.83 & 10 & $\mathrm{H}, 3-\mathrm{Br}, 4-\mathrm{Br}, 2-\mathrm{Cl}, 3-\mathrm{Cl}, 4-\mathrm{Cl}, 4-\mathrm{F}, 4-\mathrm{CH}_{3}, 3-\mathrm{NO}_{2}, 4-\mathrm{NO}_{2}$ \\
\hline & $\mathrm{F}$ & 0.809 & 756.61 & -5.340 & 14.83 & 10 & $\mathrm{H}, 3-\mathrm{Br}, 4-\mathrm{Br}, 2-\mathrm{Cl}, 3-\mathrm{Cl}, 4-\mathrm{Cl}, 4-\mathrm{F}, 4-\mathrm{CH}_{3}, 3-\mathrm{NO}_{2}, 4-\mathrm{NO}_{2}$ \\
\hline & $\mathrm{R}$ & 0.502 & 754.11 & -1.608 & 14.90 & 10 & $\mathrm{H}, 3-\mathrm{Br}, 4-\mathrm{Br}, 2-\mathrm{Cl}, 3-\mathrm{Cl}, 4-\mathrm{Cl}, 4-\mathrm{F}, 4-\mathrm{CH}_{3}, 3-\mathrm{NO}_{2}, 4-\mathrm{NO}_{2}$ \\
\hline
\end{tabular}




\begin{tabular}{|c|c|c|c|c|c|c|c|}
\hline \multirow{6}{*}{ 胥 } & $\sigma$ & 0.700 & 1035.61 & -0.275 & 13.60 & 10 & $\mathrm{H}, 3-\mathrm{Br}, 4-\mathrm{Br}, 2-\mathrm{Cl}, 3-\mathrm{Cl}, 4-\mathrm{Cl}, 4-\mathrm{F}, 4-\mathrm{CH}_{3}, 3-\mathrm{NO}_{2}, 4-\mathrm{NO}_{2}$ \\
\hline & $\sigma^{+}$ & 0.601 & 1035.41 & 0.441 & 13.60 & 10 & $\mathrm{H}, 3-\mathrm{Br}, 4-\mathrm{Br}, 2-\mathrm{Cl}, 3-\mathrm{Cl}, 4-\mathrm{Cl}, 4-\mathrm{F}, 4-\mathrm{CH}_{3}, 3-\mathrm{NO}_{2}, 4-\mathrm{NO}_{2}$ \\
\hline & $\sigma_{\mathrm{I}}$ & 0.743 & 1045.14 & -23.248 & 12.25 & 10 & $\mathrm{H}, 3-\mathrm{Br}, 4-\mathrm{Br}, 2-\mathrm{Cl}, 3-\mathrm{Cl}, 4-\mathrm{Cl}, 4-\mathrm{F}, 4-\mathrm{CH}_{3}, 3-\mathrm{NO}_{2}, 4-\mathrm{NO}_{2}$ \\
\hline & $\sigma_{\mathrm{R}}$ & 0.961 & 1040.58 & 42.062 & 10.73 & 7 & $\mathrm{H}, 3-\mathrm{Br}, 2-\mathrm{Cl}, 3-\mathrm{Cl}, 4-\mathrm{F}, 3-\mathrm{NO}_{2}, 4-\mathrm{NO}_{2}$ \\
\hline & $\mathrm{F}$ & 0.744 & 1045.25 & -23.068 & 12.17 & 10 & $\mathrm{H}, 3-\mathrm{Br}, 4-\mathrm{Br}, 2-\mathrm{Cl}, 3-\mathrm{Cl}, 4-\mathrm{Cl}, 4-\mathrm{F}, 4-\mathrm{CH}_{3}, 3-\mathrm{NO}_{2}, 4-\mathrm{NO}_{2}$ \\
\hline & $\mathrm{R}$ & 0.645 & 1040.76 & 33.295 & 11.37 & 10 & $\mathrm{H}, 3-\mathrm{Br}, 4-\mathrm{Br}, 2-\mathrm{Cl}, 3-\mathrm{Cl}, 4-\mathrm{Cl}, 4-\mathrm{F}, 4-\mathrm{CH}_{3}, 3-\mathrm{NO}_{2}, 4-\mathrm{NO}_{2}$ \\
\hline \multirow{6}{*}{$\stackrel{\overbrace{}}{\stackrel{1}{\mathscr{B}}}$} & $\sigma$ & 0.720 & 575.29 & -16.765 & 25.34 & 10 & $\mathrm{H}, 3-\mathrm{Br}, 4-\mathrm{Br}, 2-\mathrm{Cl}, 3-\mathrm{Cl}, 4-\mathrm{Cl}, 4-\mathrm{F}, 4-\mathrm{CH}_{3}, 3-\mathrm{NO}_{2}, 4-\mathrm{NO}_{2}$ \\
\hline & $\sigma^{+}$ & 0.703 & 569.80 & 2.613 & 25.89 & 10 & $\mathrm{H}, 3-\mathrm{Br}, 4-\mathrm{Br}, 2-\mathrm{Cl}, 3-\mathrm{Cl}, 4-\mathrm{Cl}, 4-\mathrm{F}, 4-\mathrm{CH}_{3}, 3-\mathrm{NO}_{2}, 4-\mathrm{NO}_{2}$ \\
\hline & $\sigma_{\mathrm{I}}$ & 0.735 & 585.36 & -35.829 & 24.25 & 10 & $\mathrm{H}, 3-\mathrm{Br}, 4-\mathrm{Br}, 2-\mathrm{Cl}, 3-\mathrm{Cl}, 4-\mathrm{Cl}, 4-\mathrm{F}, 4-\mathrm{CH}_{3}, 3-\mathrm{NO}_{2}, 4-\mathrm{NO}_{2}$ \\
\hline & $\sigma_{R}$ & 0.705 & 570.83 & 2.249 & 25.90 & 10 & $\mathrm{H}, 3-\mathrm{Br}, 4-\mathrm{Br}, 2-\mathrm{Cl}, 3-\mathrm{Cl}, 4-\mathrm{Cl}, 4-\mathrm{F}, 4-\mathrm{CH}_{3}, 3-\mathrm{NO}_{2}, 4-\mathrm{NO}_{2}$ \\
\hline & $\mathrm{F}$ & 0.746 & 589.72 & -45.506 & 22.97 & 10 & $\mathrm{H}, 3-\mathrm{Br}, 4-\mathrm{Br}, 2-\mathrm{Cl}, 3-\mathrm{Cl}, 4-\mathrm{Cl}, 4-\mathrm{F}, 4-\mathrm{CH}_{3}, 3-\mathrm{NO}_{2}, 4-\mathrm{NO}_{2}$ \\
\hline & $\mathrm{R}$ & 0.705 & 571.57 & 6.382 & 25.87 & 10 & $\mathrm{H}, 3-\mathrm{Br}, 4-\mathrm{Br}, 2-\mathrm{Cl}, 3-\mathrm{Cl}, 4-\mathrm{Cl}, 4-\mathrm{F}, 4-\mathrm{CH}_{3}, 3-\mathrm{NO}_{2}, 4-\mathrm{NO}_{2}$ \\
\hline \multirow{6}{*}{ 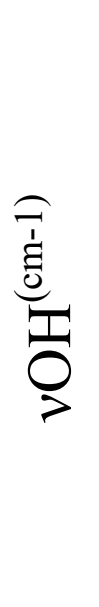 } & $\sigma$ & 0.742 & 3437.72 & 17.768 & 12.10 & 10 & $\mathrm{H}, 3-\mathrm{Br}, 4-\mathrm{Br}, 2-\mathrm{Cl}, 3-\mathrm{Cl}, 4-\mathrm{Cl}, 4-\mathrm{F}, 4-\mathrm{CH}_{3}, 3-\mathrm{NO}_{2}, 4-\mathrm{NO}_{2}$ \\
\hline & $\sigma^{+}$ & 0.740 & 3438.55 & 14.365 & 12.26 & 10 & $\mathrm{H}, 3-\mathrm{Br}, 4-\mathrm{Br}, 2-\mathrm{Cl}, 3-\mathrm{Cl}, 4-\mathrm{Cl}, 4-\mathrm{F}, 4-\mathrm{CH}_{3}, 3-\mathrm{NO}_{2}, 4-\mathrm{NO}_{2}$ \\
\hline & $\sigma_{\mathrm{I}}$ & 0.629 & 3436.39 & 15.337 & 12.79 & 10 & $\mathrm{H}, 3-\mathrm{Br}, 4-\mathrm{Br}, 2-\mathrm{Cl}, 3-\mathrm{Cl}, 4-\mathrm{Cl}, 4-\mathrm{F}, 4-\mathrm{CH}_{3}, 3-\mathrm{NO}_{2}, 4-\mathrm{NO}_{2}$ \\
\hline & $\sigma_{\mathrm{R}}$ & 0.643 & 3446.24 & 29.244 & 12.05 & 10 & $\mathrm{H}, 3-\mathrm{Br}, 4-\mathrm{Br}, 2-\mathrm{Cl}, 3-\mathrm{Cl}, 4-\mathrm{Cl}, 4-\mathrm{F}, 4-\mathrm{CH}_{3}, 3-\mathrm{NO}_{2}, 4-\mathrm{NO}_{2}$ \\
\hline & $\mathrm{F}$ & 0.718 & 3438.75 & 9.439 & 13.14 & 10 & $\mathrm{H}, 3-\mathrm{Br}, 4-\mathrm{Br}, 2-\mathrm{Cl}, 3-\mathrm{Cl}, 4-\mathrm{Cl}, 4-\mathrm{F}, 4-\mathrm{CH}_{3}, 3-\mathrm{NO}_{2}, 4-\mathrm{NO}_{2}$ \\
\hline & $\mathrm{R}$ & 0.634 & 3446.81 & 25.970 & 12.05 & 10 & $\mathrm{H}, 3-\mathrm{Br}, 4-\mathrm{Br}, 2-\mathrm{Cl}, 3-\mathrm{Cl}, 4-\mathrm{Cl}, 4-\mathrm{F}, 4-\mathrm{CH}_{3}, 3-\mathrm{NO}_{2}, 4-\mathrm{NO}_{2}$ \\
\hline
\end{tabular}




\begin{tabular}{|c|c|c|c|c|c|c|c|}
\hline \multirow{6}{*}{ 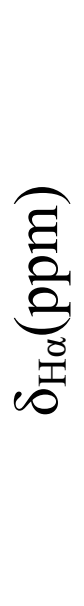 } & $\sigma$ & 0.914 & 7.468 & 0.457 & 0.06 & 10 & $\mathrm{H}, 3-\mathrm{Br}, 4-\mathrm{Br}, 2-\mathrm{Cl}, 3-\mathrm{Cl}, 4-\mathrm{Cl}, 4-\mathrm{F}, 4-\mathrm{CH}_{3}, 3-\mathrm{NO}_{2}, 4-\mathrm{NO}_{2}$ \\
\hline & $\sigma^{+}$ & 0.905 & 7.490 & 0.367 & 0.08 & 10 & $\mathrm{H}, 3-\mathrm{Br}, 4-\mathrm{Br}, 2-\mathrm{Cl}, 3-\mathrm{Cl}, 4-\mathrm{Cl}, 4-\mathrm{F}, 4-\mathrm{CH}_{3}, 3-\mathrm{NO}_{2}, 4-\mathrm{NO}_{2}$ \\
\hline & $\sigma_{\mathrm{I}}$ & 0.638 & 7.432 & 0.398 & 0.12 & 10 & $\mathrm{H}, 3-\mathrm{Br}, 4-\mathrm{Br}, 2-\mathrm{Cl}, 3-\mathrm{Cl}, 4-\mathrm{Cl}, 4-\mathrm{F}, 4-\mathrm{CH}_{3}, 3-\mathrm{NO}_{2}, 4-\mathrm{NO}_{2}$ \\
\hline & $\sigma_{\mathrm{R}}$ & 0.730 & 7.668 & 0.590 & 0.10 & 10 & $\mathrm{H}, 3-\mathrm{Br}, 4-\mathrm{Br}, 2-\mathrm{Cl}, 3-\mathrm{Cl}, 4-\mathrm{Cl}, 4-\mathrm{F}, 4-\mathrm{CH}_{3}, 3-\mathrm{NO}_{2}, 4-\mathrm{NO}_{2}$ \\
\hline & $\mathrm{F}$ & 0.544 & 7.457 & 0.332 & 0.13 & 10 & $\mathrm{H}, 3-\mathrm{Br}, 4-\mathrm{Br}, 2-\mathrm{Cl}, 3-\mathrm{Cl}, 4-\mathrm{Cl}, 4-\mathrm{F}, 4-\mathrm{CH}_{3}, 3-\mathrm{NO}_{2}, 4-\mathrm{NO}_{2}$ \\
\hline & $\mathrm{R}$ & 0.728 & 7.678 & 0.518 & 0.11 & 10 & $\mathrm{H}, 3-\mathrm{Br}, 4-\mathrm{Br}, 2-\mathrm{Cl}, 3-\mathrm{Cl}, 4-\mathrm{Cl}, 4-\mathrm{F}, 4-\mathrm{CH}_{3}, 3-\mathrm{NO}_{2}, 4-\mathrm{NO}_{2}$ \\
\hline \multirow{6}{*}{ 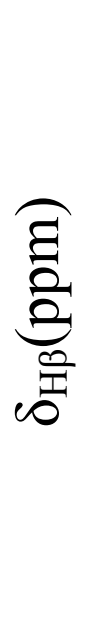 } & $\sigma$ & 0.726 & 7.935 & 0.165 & 0.19 & 10 & $\mathrm{H}, 3-\mathrm{Br}, 4-\mathrm{Br}, 2-\mathrm{Cl}, 3-\mathrm{Cl}, 4-\mathrm{Cl}, 4-\mathrm{F}, 4-\mathrm{CH}_{3}, 3-\mathrm{NO}_{2}, 4-\mathrm{NO}_{2}$ \\
\hline & $\sigma^{+}$ & 0.947 & 7.907 & 0.255 & 0.17 & 8 & $\mathrm{H}, 3-\mathrm{Br}, 4-\mathrm{Br}, 3-\mathrm{Cl}, 4-\mathrm{Cl}, 4-\mathrm{F}, 3-\mathrm{NO}_{2}, 4-\mathrm{NO}_{2}$ \\
\hline & $\sigma_{\mathrm{I}}$ & 0.420 & 7.846 & 0.328 & 0.18 & 10 & $\mathrm{H}, 3-\mathrm{Br}, 4-\mathrm{Br}, 2-\mathrm{Cl}, 3-\mathrm{Cl}, 4-\mathrm{Cl}, 4-\mathrm{F}, 4-\mathrm{CH}_{3}, 3-\mathrm{NO}_{2}, 4-\mathrm{NO}_{2}$ \\
\hline & $\sigma_{\mathrm{R}}$ & 0.707 & 7.972 & -0.079 & 0.19 & 10 & $\mathrm{H}, 3-\mathrm{Br}, 4-\mathrm{Br}, 2-\mathrm{Cl}, 3-\mathrm{Cl}, 4-\mathrm{Cl}, 4-\mathrm{F}, 4-\mathrm{CH}_{3}, 3-\mathrm{NO}_{2}, 4-\mathrm{NO}_{2}$ \\
\hline & $\mathrm{F}$ & 0.732 & 7.877 & 0.248 & 0.18 & 10 & $\mathrm{H}, 3-\mathrm{Br}, 4-\mathrm{Br}, 2-\mathrm{Cl}, 3-\mathrm{Cl}, 4-\mathrm{Cl}, 4-\mathrm{F}, 4-\mathrm{CH}_{3}, 3-\mathrm{NO}_{2}, 4-\mathrm{NO}_{2}$ \\
\hline & $\mathrm{R}$ & 0.704 & 7.988 & 0.036 & 0.19 & 10 & $\mathrm{H}, 3-\mathrm{Br}, 4-\mathrm{Br}, 2-\mathrm{Cl}, 3-\mathrm{Cl}, 4-\mathrm{Cl}, 4-\mathrm{F}, 4-\mathrm{CH}_{3}, 3-\mathrm{NO}_{2}, 4-\mathrm{NO}_{2}$ \\
\hline \multirow{6}{*}{$\overbrace{0}^{\frac{8}{2}}$} & $\sigma$ & 0.838 & 192.54 & 0.582 & 0.44 & 10 & $\mathrm{H}, 3-\mathrm{Br}, 4-\mathrm{Br}, 2-\mathrm{Cl}, 3-\mathrm{Cl}, 4-\mathrm{Cl}, 4-\mathrm{F}, 4-\mathrm{CH}_{3}, 3-\mathrm{NO}_{2}, 4-\mathrm{NO}_{2}$ \\
\hline & $\sigma^{+}$ & 0.749 & 192.52 & 0.641 & 0.42 & 10 & $\mathrm{H}, 3-\mathrm{Br}, 4-\mathrm{Br}, 2-\mathrm{Cl}, 3-\mathrm{Cl}, 4-\mathrm{Cl}, 4-\mathrm{F}, 4-\mathrm{CH}_{3}, 3-\mathrm{NO}_{2}, 4-\mathrm{NO}_{2}$ \\
\hline & $\sigma_{\mathrm{I}}$ & 0.723 & 192.52 & 0.444 & 0.47 & 10 & $\mathrm{H}, 3-\mathrm{Br}, 4-\mathrm{Br}, 2-\mathrm{Cl}, 3-\mathrm{Cl}, 4-\mathrm{Cl}, 4-\mathrm{F}, 4-\mathrm{CH}_{3}, 3-\mathrm{NO}_{2}, 4-\mathrm{NO}_{2}$ \\
\hline & $\sigma_{\mathrm{R}}$ & 0.735 & 192.81 & 0.878 & 0.45 & 10 & $\mathrm{H}, 3-\mathrm{Br}, 4-\mathrm{Br}, 2-\mathrm{Cl}, 3-\mathrm{Cl}, 4-\mathrm{Cl}, 4-\mathrm{F}, 4-\mathrm{CH}_{3}, 3-\mathrm{NO}_{2}, 4-\mathrm{NO}_{2}$ \\
\hline & $\mathrm{F}$ & 0.760 & 192.58 & 0.296 & 0.48 & 10 & $\mathrm{H}, 3-\mathrm{Br}, 4-\mathrm{Br}, 2-\mathrm{Cl}, 3-\mathrm{Cl}, 4-\mathrm{Cl}, 4-\mathrm{F}, 4-\mathrm{CH}_{3}, 3-\mathrm{NO}_{2}, 4-\mathrm{NO}_{2}$ \\
\hline & $\mathrm{R}$ & 0.741 & 192.85 & 0.904 & 0.44 & 10 & $\mathrm{H}, 3-\mathrm{Br}, 4-\mathrm{Br}, 2-\mathrm{Cl}, 3-\mathrm{Cl}, 4-\mathrm{Cl}, 4-\mathrm{F}, 4-\mathrm{CH}_{3}, 3-\mathrm{NO}_{2}, 4-\mathrm{NO}_{2}$ \\
\hline
\end{tabular}




\begin{tabular}{|c|c|c|c|c|c|c|c|}
\hline \multirow{6}{*}{ है } & $\sigma$ & 0.728 & 122.95 & 2.920 & 4.40 & 10 & $\mathrm{H}, 3-\mathrm{Br}, 4-\mathrm{Br}, 2-\mathrm{Cl}, 3-\mathrm{Cl}, 4-\mathrm{Cl}, 4-\mathrm{F}, 4-\mathrm{CH}_{3}, 3-\mathrm{NO}_{2}, 4-\mathrm{NO}_{2}$ \\
\hline & $\sigma^{+}$ & 0.829 & 122.75 & 3.515 & 4.30 & 10 & $\mathrm{H}, 3-\mathrm{Br}, 4-\mathrm{Br}, 2-\mathrm{Cl}, 3-\mathrm{Cl}, 4-\mathrm{Cl}, 4-\mathrm{F}, 4-\mathrm{CH}_{3}, 3-\mathrm{NO}_{2}, 4-\mathrm{NO}_{2}$ \\
\hline & $\sigma_{\mathrm{I}}$ & 0.634 & 120.58 & 7.726 & 4.04 & 10 & $\mathrm{H}, 3-\mathrm{Br}, 4-\mathrm{Br}, 2-\mathrm{Cl}, 3-\mathrm{Cl}, 4-\mathrm{Cl}, 4-\mathrm{F}, 4-\mathrm{CH}_{3}, 3-\mathrm{NO}_{2}, 4-\mathrm{NO}_{2}$ \\
\hline & $\sigma_{R}$ & 0.731 & 122.91 & -7.169 & 4.26 & 10 & $\mathrm{H}, 3-\mathrm{Br}, 4-\mathrm{Br}, 2-\mathrm{Cl}, 3-\mathrm{Cl}, 4-\mathrm{Cl}, 4-\mathrm{F}, 4-\mathrm{CH}_{3}, 3-\mathrm{NO}_{2}, 4-\mathrm{NO}_{2}$ \\
\hline & $\mathrm{F}$ & 0.745 & 120.47 & 7.836 & 3.99 & 10 & $\mathrm{H}, 3-\mathrm{Br}, 4-\mathrm{Br}, 2-\mathrm{Cl}, 3-\mathrm{Cl}, 4-\mathrm{Cl}, 4-\mathrm{F}, 4-\mathrm{CH}_{3}, 3-\mathrm{NO}_{2}, 4-\mathrm{NO}_{2}$ \\
\hline & $\mathrm{R}$ & 0.730 & 122.80 & -6.197 & 4.28 & 10 & $\mathrm{H}, 3-\mathrm{Br}, 4-\mathrm{Br}, 2-\mathrm{Cl}, 3-\mathrm{Cl}, 4-\mathrm{Cl}, 4-\mathrm{F}, 4-\mathrm{CH}_{3}, 3-\mathrm{NO}_{2}, 4-\mathrm{NO}_{2}$ \\
\hline & $\sigma$ & 0.711 & 145.33 & 1.274 & 3.54 & 10 & $\mathrm{H}, 3-\mathrm{Br}, 4-\mathrm{Br}, 2-\mathrm{Cl}, 3-\mathrm{Cl}, 4-\mathrm{Cl}, 4-\mathrm{F}, 4-\mathrm{CH}_{3}, 3-\mathrm{NO}_{2}, 4-\mathrm{NO}_{2}$ \\
\hline & $\sigma^{+}$ & 0.713 & 145.30 & 1.319 & 3.52 & 10 & $\mathrm{H}, 3-\mathrm{Br}, 4-\mathrm{Br}, 2-\mathrm{Cl}, 3-\mathrm{Cl}, 4-\mathrm{Cl}, 4-\mathrm{F}, 4-\mathrm{CH}_{3}, 3-\mathrm{NO}_{2}, 4-\mathrm{NO}_{2}$ \\
\hline & $\sigma_{\mathrm{I}}$ & 0.808 & 145.20 & 1.177 & 3.55 & 10 & $\mathrm{H}, 3-\mathrm{Br}, 4-\mathrm{Br}, 2-\mathrm{Cl}, 3-\mathrm{Cl}, 4-\mathrm{Cl}, 4-\mathrm{F}, 4-\mathrm{CH}_{3}, 3-\mathrm{NO}_{2}, 4-\mathrm{NO}_{2}$ \\
\hline & $\sigma_{\mathrm{R}}$ & 0.808 & 145.88 & 1.607 & 3.54 & 10 & $\mathrm{H}, 3-\mathrm{Br}, 4-\mathrm{Br}, 2-\mathrm{Cl}, 3-\mathrm{Cl}, 4-\mathrm{Cl}, 4-\mathrm{F}, 4-\mathrm{CH}_{3}, 3-\mathrm{NO}_{2}, 4-\mathrm{NO}_{2}$ \\
\hline & $\mathrm{F}$ & 0.810 & 145.18 & 1.196 & 3.55 & 10 & $\mathrm{H}, 3-\mathrm{Br}, 4-\mathrm{Br}, 2-\mathrm{Cl}, 3-\mathrm{Cl}, 4-\mathrm{Cl}, 4-\mathrm{F}, 4-\mathrm{CH}_{3}, 3-\mathrm{NO}_{2}, 4-\mathrm{NO}_{2}$ \\
\hline & $\mathrm{R}$ & 0.712 & 146.01 & 2.046 & 3.53 & 10 & $\mathrm{H}, 3-\mathrm{Br}, 4-\mathrm{Br}, 2-\mathrm{Cl}, 3-\mathrm{Cl}, 4-\mathrm{Cl}, 4-\mathrm{F}, 4-\mathrm{CH}_{3}, 3-\mathrm{NO}_{2}, 4-\mathrm{NO}_{2}$ \\
\hline
\end{tabular}

$\mathbf{r}=$ Correlation co-efficient; $\boldsymbol{\rho}=$ Slope $\mathbf{I}=$ Intercept $; \mathbf{s}=$ Standard deviation; $\mathbf{n}=$ Number of substituents.

The inability of effect of substituents on absorption and the resonance conjugative structure shown in Fig. 2. regression analysis of these frequencies of all ketones with inductive, resonance and Swain - Lupton's [22] constants produce satisfactory correlations as evident in equations 2 and 3.

$$
\begin{gathered}
\lambda_{(\mathrm{nm})}=320.28( \pm 18.167)-45.151( \pm 36.359) \sigma_{\mathrm{I}}-26.949( \pm 46.585) \sigma_{\mathrm{R}} \\
(R=0.946, \mathrm{n}=10, P>90 \%) \\
\lambda_{(\mathrm{nm})}=313.31( \pm 18.011)-34.042( \pm 36.579) \mathrm{F}-37.516( \pm 42.981) \mathrm{R} \\
(R=0.941, \mathrm{n}=10, P>90 \%)
\end{gathered}
$$




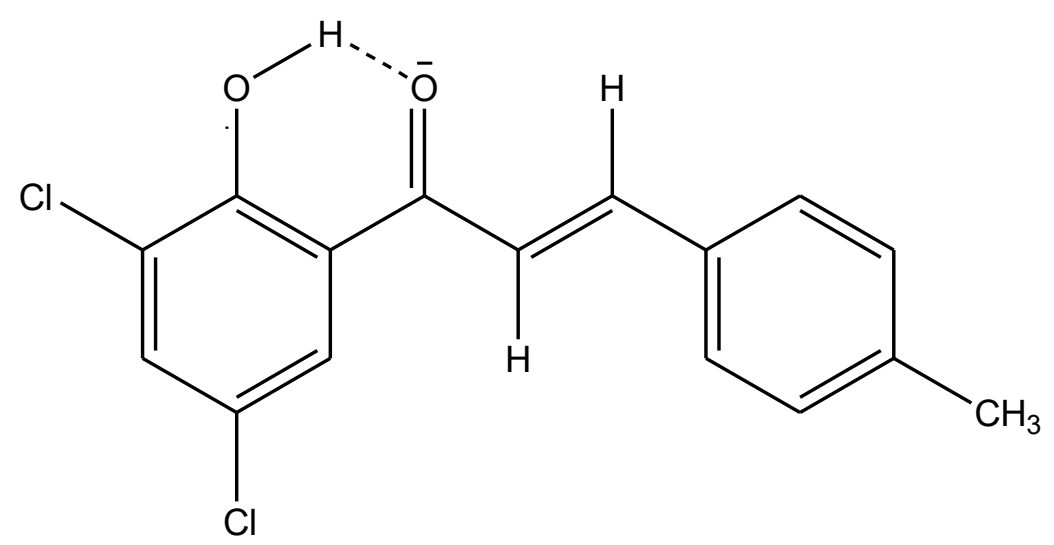

Fig. 2. The resonance-conjugative structure

\section{2. IR spectral study}

The carbonyl stretching frequencies $\left(\mathrm{cm}^{-1}\right)$ of $s$-cis and s-trans isomers of present study are presented in Table 2. The stretching frequencies for carbonyl absorption are assigned based on the assignments made by Hays and Timmons [23] for $s$-cis and s-trans conformers at 1690 and $1670 \mathrm{~cm}^{-1}$, respectively. As anticipated the lowest carbonyl frequency is observed in both the conformers when strongest electron withdrawing group is present in phenyl ring while highest frequency is noted when strongest electro attracting group present in phenyl ring. A similarly trend in absorption was earlier noted by Perjessy and Hrnciar [24] too whose investigated on chalcones demonstrates that $s$-trans conformers transmit more effectively than $s$-cis conformers due to reason stated earlier. The difference in carbonyl frequencies between the $s$-cis and s-trans conformers is higher in this study than the difference observed by Silver and Boykin [25] between similar conformers in phenyl styryl ketones. These data have been correlated with Hammett substituent constants and Swain-Lupton constants [22]. In this correlation the structure parameter Hammett equation employed is as shown in the following equation:

$$
v=\rho \sigma+v_{0}
$$

Where $v$ is the carbonyl frequencies of substituted system and $v_{0}$ is the corresponding quantity of unsubstitued system; $\sigma$ is a Hammett substituent constant, which in principle is characteristics of the substituent and $\rho$ is a reaction constant which is depend upon the nature of the reaction. Hammett equation is one of the important tools for studying linear free energy relationships and it has been widely used in structures of the chemical reactivity of substituted aromatic system.

From Table 2, the s-cis conformers gave satisfactory correlation with Hammett $\sigma, \sigma^{+}$, and $\sigma_{\mathrm{I}}$ constants. And the s-trans conformers, the correlation $v_{\mathrm{C}=\mathrm{O}}$ fails with Hammett $\sigma$ parameters. All correlations gave positive $\rho$ values and it implies that there is a normal substituent effects operates in all systems.

The correlation of $\mathrm{CH}$ in-plane and out of plane modes with Hammett $\sigma$ constants were fails in correlation. The $\mathrm{CH}$ in-plane modes gave negative $\rho$ values in all correlations.

A satisfactory correlation obtained for $\mathrm{CH}=\mathrm{CH}$ out of plane modes with Hammett $\sigma_{\mathrm{R}}$ constants. Also all correlation were fails with $\mathrm{C}=\mathrm{C}$ out of plane modes with Hammett constants. 
Similarly the $\mathrm{vOH}$ stretches also fail in correlations. This is due to the inability polar, resonance and inductive effects substituent constant to predict the reactivity of the frequencies along with the resonance conjugative structure shown in Fig. 2. The correlation of $v \mathrm{OH}$ stretches were fails in correlation with Hammett substituent constants, F and R parameters..

Some of the individual single parameter correlations were fails with hammett substituent constants and $\mathrm{F}$ and $\mathrm{R}$ parameters. While the multi-regression analysis seems worthwhile with Swain- Luptons[22] constants and the generated equations are shown in 5-18.

$$
\begin{gathered}
\mathrm{vCO}_{\text {s-cis }}\left(\mathrm{cm}^{-1}\right)=1638.33( \pm 1.996)+14.926( \pm 3.994) \sigma_{\mathrm{I}}+4.105( \pm 5.118) \sigma_{\mathrm{R}} \\
(R=0.982, \mathrm{n}=10, P>95 \%) \\
\mathrm{vCO}_{\text {s-cis }}\left(\mathrm{cm}^{-1}\right)=1638.69( \pm 1.904)+14.813( \pm 3.868) \mathrm{F}+5.864( \pm 4.545) \mathrm{R} \\
(R=0.982, \mathrm{n}=10, P>95 \%) \\
v \mathrm{CO}_{\text {s-trans }}\left(\mathrm{cm}^{-1}\right)=1580.47( \pm 4.533)+4.359( \pm 9.073) \sigma_{\mathrm{I}}+3.073( \pm 11.624) \sigma_{\mathrm{R}} \\
(R=0.920, \mathrm{n}=10, P>90 \%) \\
v \mathrm{CO}_{\text {s-trans }}\left(\mathrm{cm}^{-1}\right)=1579.90( \pm 4.332)+5.610( \pm 8.798) \mathrm{F}+2.313( \pm 10.338) \mathrm{R} \\
(R=0.923, \mathrm{n}=10, P>90 \%)
\end{gathered}
$$

$$
\begin{gathered}
\mathrm{CCH}_{i p}\left(\mathrm{~cm}^{-1}\right)=1169.25( \pm 7.919)-20.819( \pm 16.083) \mathrm{F}+1.511( \pm 18.898) \mathrm{R} \\
(R=0.944, \mathrm{n}=10, P>90 \%)
\end{gathered}
$$$$
v \mathrm{CH}_{o p}\left(\mathrm{~cm}^{-1}\right)=754.90( \pm 10.995)-3.422( \pm 22.004) \sigma_{\mathrm{I}}-7.287( \pm 28.193) \sigma_{\mathrm{R}}
$$$$
(R=0.911, \mathrm{n}=10, P>90 \%)
$$

$$
\begin{gathered}
\mathrm{vCH}_{o p}\left(\mathrm{~cm}^{-1}\right)=756.34( \pm 10.604)-5.667( \pm 21.536) \mathrm{F}-2.596( \pm 25.305) \mathrm{R} \\
(R=0.910, \mathrm{n}=10, P>90 \%)
\end{gathered}
$$

$$
\begin{gathered}
v \mathrm{CH}=\mathrm{CH}_{o p}\left(\mathrm{~cm}^{-1}\right)=1050.69( \pm 6.525)-24.218( \pm 13.058) \sigma_{\mathrm{I}}+42.959( \pm 16.731) \sigma_{\mathrm{R}} \\
(R=0.976, \mathrm{n}=10, P>95 \%)
\end{gathered}
$$

$v \mathrm{CH}=\mathrm{CH}_{o p}\left(\mathrm{~cm}^{-1}\right)=1048.36( \pm 7.297)-19.288( \pm 14.821) \mathrm{F}+29.932( \pm 17.415) \mathrm{R}$

$$
(R=0.966, \mathrm{n}=10, P>95 \%)
$$$$
{ } \mathrm{C}=\mathrm{C}_{o p}\left(\mathrm{~cm}^{-1}\right)=585.82( \pm 17.994)-35.910( \pm 36.012) \sigma_{\mathrm{I}}+3.579( \pm 46.140) \sigma_{\mathrm{R}}
$$

$$
(R=0.935, \mathrm{n}=10, P>90 \%)
$$

$$
\begin{gathered}
v \mathrm{C}=\mathrm{C}_{o p}\left(\mathrm{~cm}^{-1}\right)=589.56( \pm 16.422)-45.707( \pm 33.352) \mathrm{F}-1.587( \pm 39.189) \mathrm{R} \\
(R=0.946, \mathrm{n}=10, P>90 \%)
\end{gathered}
$$

$$
\begin{array}{cc}
\mathrm{vOH}\left(\mathrm{cm}^{-1}\right) & =3440.11( \pm 8.506)+14.690( \pm 17.023) \sigma \mathrm{I}+28.700( \pm 21.811) \sigma_{\mathrm{R}} \\
& (R=0.951, \mathrm{n}=10, P>95 \%) \\
v \mathrm{OH}\left(\mathrm{cm}^{-1}\right) & =3441.69( \pm 8.267)+13.006( \pm 16.789) \mathrm{F}+28.238( \pm 19.728) \mathrm{R} \\
(R=0.940, \mathrm{n}=10, \mathrm{P}>90 \%)
\end{array}
$$




\section{3. ${ }^{1} \mathrm{H}$ NMR spectral correlation}

In the present study the ${ }^{1} \mathrm{H}$ NMR spectra of the chalcones under investigation have been recorded in deuterated chloroform employing tetramethylsilane (TMS) as internal standard. The signals of the ethylenic protons have been assigned. They are calculated as $A B$ or AA' BB' systems respectively $[26,27]$. The chemical shifts of $H_{\alpha}$ are found at higher field than those of $\mathrm{H}_{\beta}$ in this series of ketones. The ethylenic protons give an $\mathrm{AB}$ pattern and the $\beta-$ proton doublet in most cases is well separated from the signals of the aromatic protons. The assigned chemical shifts of the ethylenic protons are presented in Table 2.

In nuclear magnetic resonance spectra, the proton chemical shift $\delta(\mathrm{ppm})$ depends on the electronic environment of the nuclei concerned. These shifts have been correlated with reactivity parameters. Thus the Hammett equation employed for the analysis in the form as is as shown below:

$$
\log \delta=\log \delta_{0}+\rho \sigma
$$

where $\delta_{0}$ is the chemical shift in the corresponding parent compound.

The assigned ${ }^{1} \mathrm{H}$ NMR chemical shifts $(\delta \mathrm{ppm})$ of $\mathrm{H}_{\alpha}$ and $\mathrm{H}_{\beta}$ of substituted styryl 3,5duchloro-2-hydroxyphenyl ketones are presented in Table 2. These chemical shifts are correlated with Hammett substituent constants and $\mathrm{F}$ and $\mathrm{R}$ parameters. The results of statistical analysis these chemical shifts (ppm) are shown in Table 3. Hammett $\sigma$ and $\sigma^{+}$, parameters gave satisfactory correlations with $\delta \mathrm{H}_{\alpha}$ chemical shifts with positive $\rho$ values. The $\delta \mathrm{H}_{\beta}$ chemical shifts gave satisfactory correlation with Hammett $\sigma^{+}$constant. The remaining Hammett substituent constants, $\mathrm{F}$ and $\mathrm{R}$ parameters were fail correlations. This is due to the reasons stated earlier and associated with the resonance conjugative structure. The degree of transmission of substituent effects on $\delta \mathrm{H}_{\alpha}$ is higher than $\delta \mathrm{H}_{\beta}$ chemical shifts.

Some of the single parameter correlations of $\delta \mathrm{H}_{\alpha}$ and $\delta \mathrm{H}_{\beta}$ chemical shifts fail with Hammett sigma constants and $\mathrm{F}$ and $\mathrm{R}$ parameters. While seeking the multi regression analysis, there is satisfactorily correlations observed with Swain-Lupton and F and R parameters[22]. The correlation multi regression equations are shown below (20-23)

$$
\begin{gathered}
\delta \mathrm{H}_{\alpha}(\mathrm{ppm})=7.507( \pm 0.036)+0.385( \pm 0.073) \sigma_{\mathrm{I}}+0.576( \pm 0.094) \sigma_{\mathrm{R}} \\
(R=0.951, \mathrm{n}=10, P>95 \%) \\
\delta \mathrm{H}_{\alpha}(\mathrm{ppm})=7.578( \pm 0.023)+0.406( \pm 0.048) \mathrm{F}+0.589( \pm 0.057) \mathrm{R} \\
(R=0.978, \mathrm{n}=10, P>95 \%) \\
\delta \mathrm{H}_{\beta}(\mathrm{ppm})=7.834( \pm 0.133)+0.330( \pm 0.267) \sigma_{\mathrm{I}}-0.092( \pm 0.342) \sigma_{\mathrm{R}} \\
(R=0.943, \mathrm{n}=10, P>90 \%) \\
\delta \mathrm{H}_{\beta}(\mathrm{ppm})=7.886( \pm 0.133)+0.258( \pm 0.271) \mathrm{F}+0.081( \pm 0.319) \mathrm{R} \\
(R=0.934, \mathrm{n}=10, P>90 \%)
\end{gathered}
$$

\section{4. ${ }^{13} \mathrm{C}$ NMR spectral correlation}

The assigned carbonyl carbon chemical shifts ( $\delta \mathrm{ppm})$ of $\mathrm{CO}, \mathrm{C}_{\alpha}$ and $\mathrm{C}_{\beta}$ of 3,4-dichloro2-hydroxyphenyl chalcones are presented in Table 2 and these chemical shifts are correlated with Hammett sigma constants and $\mathrm{F}$ and $\mathrm{R}$ parameters. 
The results of statistical analysis are shown in Table 3. From the table, the $\mathrm{CO}, \mathrm{C}_{\alpha}$ and $\mathrm{C}_{\beta}$ carbon chemical shifts are found to show poor correlation with Hammett $\sigma$ constants, $\mathrm{F}$ and R parameters along with positive $\rho$ values.

This shows that the normal substituent effect gets reversed in all ketones. The polar, resonance and Field effects of the substituents also fail in correlation. This is due to the reason stated earlier and it is attributed to conjugated structure shown in Figure 2.

Single parameter correlations of the chemical shifts of ${ }^{13} \mathrm{C} N M R$ of $\mathrm{C}_{\alpha}$ and $\mathrm{C}_{\beta}(\delta \mathrm{ppm})$ from ${ }^{13} \mathrm{C}$ NMR spectral data with Hammett sigma constants and $\mathrm{F}$ and $\mathrm{R}$ parameters fail. While seeking the multi regression analysis, there is a satisfactorily correlations observed with Swain-Lupton and $\mathrm{F}$ and $\mathrm{R}$ parameters. The correlated multi regression equations are given in 24-29.

$$
\begin{gathered}
\delta \mathrm{CO}(\mathrm{ppm})=192.64( \pm 0.327)+0.424( \pm 0.655) \sigma_{\mathrm{I}}+0.863( \pm 0.840) \sigma_{\mathrm{R}} \\
(R=0.942, \mathrm{n}=10, P>90 \%) \\
\delta \mathrm{CO}(\mathrm{ppm})=192.68( \pm 0.306)+0.420( \pm 0.622) \mathrm{F}+0.977( \pm 0.731) \mathrm{R} \\
(R=0.947, \mathrm{n}=10, P>90 \%) \\
\delta \mathrm{C}_{\alpha}(\mathrm{ppm})=119.62( \pm 2.795)+7.895( \pm 5.593) \sigma_{\mathrm{I}}-7.461( \pm 7.167) \sigma_{\mathrm{R}} \\
(R=0.954, \mathrm{n}=10, P>95 \%) \\
\delta \mathrm{C}_{\alpha}(\mathrm{ppm})=119.96( \pm 2.749)+7.212( \pm 5.583) \mathrm{F}-4.939( \pm 6.560) \mathrm{R} \\
(R=0.951, \mathrm{n}=10, P>95 \%) \\
\delta \mathrm{C}_{\beta}(\mathrm{ppm})=145.40( \pm 2.658)+1.142( \pm 5.254) \sigma_{\mathrm{I}}+1.564( \pm 6.732) \sigma_{\mathrm{R}} \\
(R=0.912, \mathrm{n}=10, P>90 \%) \\
\delta \mathrm{C}_{\beta}(\mathrm{ppm})=145.42( \pm 2.511)+1.487( \pm 5.100) \mathrm{F}+2.305( \pm 5.992) \mathrm{R} \\
(R=0.916, \mathrm{n}=10, P>90 \%)
\end{gathered}
$$

\section{5. Antimicrobial activities}

Chalcones possess a wide range of biological activities such as antibacterial [7, 8], antifungal [9], antiviral [11], antifeedant [19], antimalarial [12], antituberculosis [14], and antioxidant [10] activities. These multipronged activities present in different chalcones are examined against respective microbes-bacteria's.

\section{5. 1. Antibacterial sensitivity assay}

Antibacterial sensitivity assay was performed using Kirby-Bauer [28] disc diffusion technique. In each Petri plate about $0.5 \mathrm{ml}$ of the test bacterial sample was spread uniformly over the solidified Mueller Hinton agar using sterile glass spreader. Then the discs with $5 \mathrm{~mm}$ diameter made up of Whatman No.1 filter paper, impregnated with the solution of the compound were placed on the medium using sterile forceps. The plates were incubated for 24 hours at $37{ }^{\circ} \mathrm{C}$ by keeping the plates upside down to prevent the collection of water droplets over the medium. After 24 hours, the plates were visually examined and the diameter values of the zone of inhibition were measured. Triplicate results were recorded by repeating the same procedure. 
The antibacterial screening effect of synthesized chalcones is shown in Figure 4. (Plates 1-10). The zone of inhibition is compared using Table 4 and the Clustered column Chart is shown in Figure 5. A good antibacterial activity was possessed by all substituents on the microorganisms in general. All the compounds showed excellent activities on B.subtilis and P.aeruginosa species. The substituent $4-\mathrm{Cl}, 4-\mathrm{CH}_{3}$ and $3-\mathrm{NO}_{2}$ have high activity against B.subtilis. The substituent 4-Br and 4-Cl have high activity against E.Coli. The substituent 3$\mathrm{NO}_{2}$ has improved antibacterial activity against S.aureus than other species.

Table 4. Antibacterial activity of 3,5-dichloro-2-hydroxyphenyl chalcones(entries 27-36).

\begin{tabular}{|c|c|c|c|c|c|c|}
\hline \multirow{3}{*}{ Entry } & \multirow{3}{*}{ Substt. } & \multicolumn{5}{|c|}{ Zone of Inhibition (mm) } \\
\hline & & \multicolumn{3}{|c|}{ Gram positive Bacteria } & \multicolumn{2}{|c|}{ Gram negative Bacteria } \\
\hline & & B.subtilis & M.luteus & S.aureus & E.coli & P.aeruginosa \\
\hline 27 & $\mathrm{H}$ & 6 & 7 & 7 & 6 & 6 \\
\hline 28 & $3-\mathrm{Br}$ & 6 & 7 & 6 & 6 & 6 \\
\hline 29 & $4-\mathrm{Br}$ & 6 & 8 & 6 & 8 & 8 \\
\hline 30 & $2-\mathrm{Cl}$ & 6 & 6 & 6 & 7 & 6 \\
\hline 31 & $3-\mathrm{Cl}$ & 6 & 6 & - & 7 & 6 \\
\hline 32 & $4-\mathrm{Cl}$ & 7 & 6 & 6 & 8 & 6 \\
\hline 33 & $4-\mathrm{F}$ & 6 & 6 & 7 & 6 & 7 \\
\hline 34 & 4-Me & 7 & 6 & 6 & 6 & 7 \\
\hline 35 & $3-\mathrm{NO}_{2}$ & 9 & 6 & 9 & 7 & 6 \\
\hline 36 & $4-\mathrm{NO}_{2}$ & 6 & 7 & 7 & 7 & 7 \\
\hline Standard & Ampicillin & 8 & 8 & 7 & 9 & 9 \\
\hline Control & DMSO & - & - & - & - & - \\
\hline
\end{tabular}




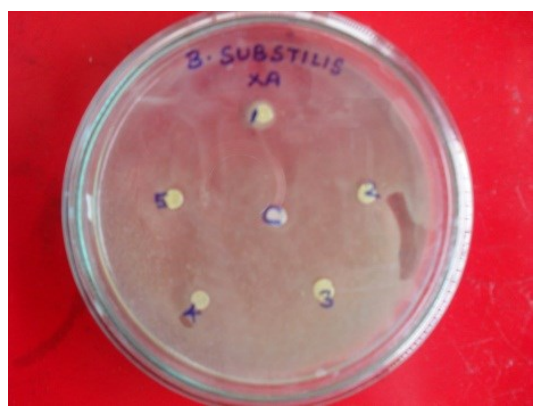

Plate-1

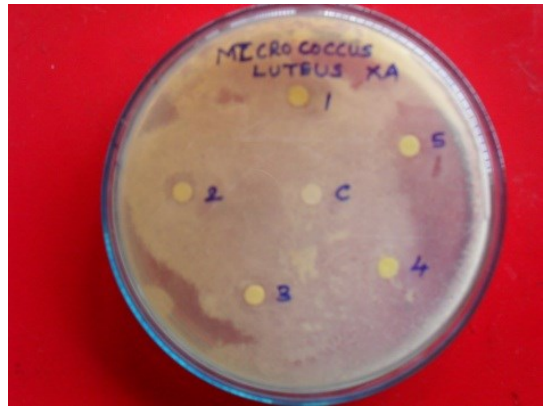

Plate-3

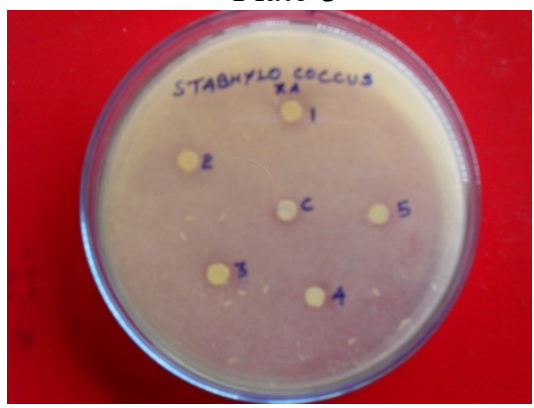

Plate-5

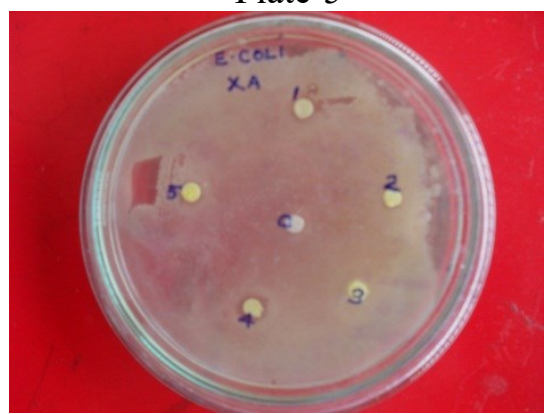

Plate-7

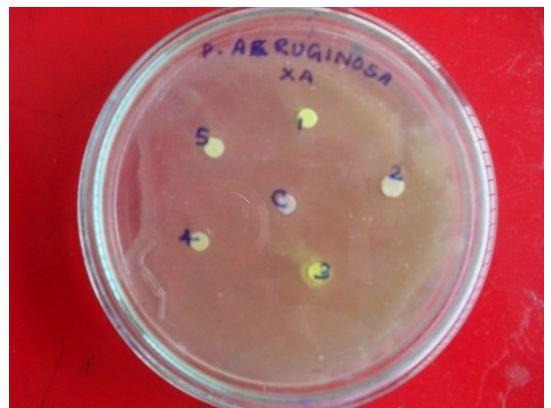

Plate-9

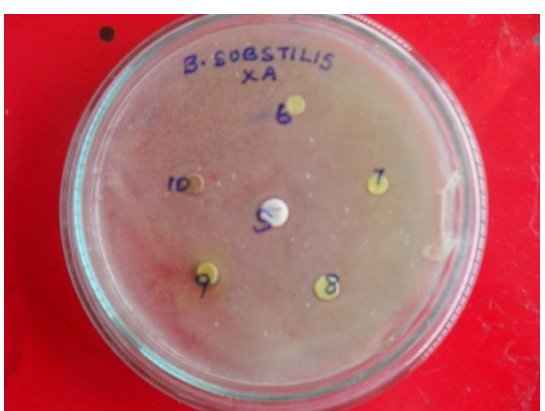

Plate-2

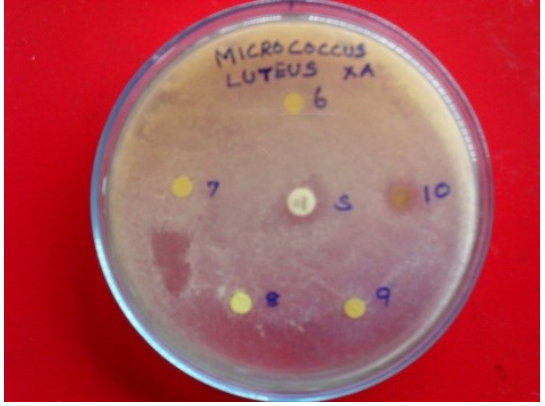

Plate-4

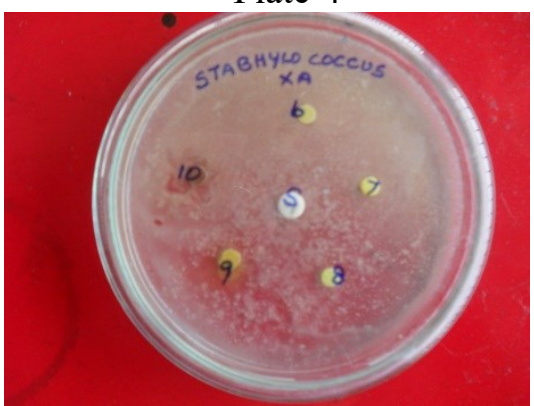

Plate-6

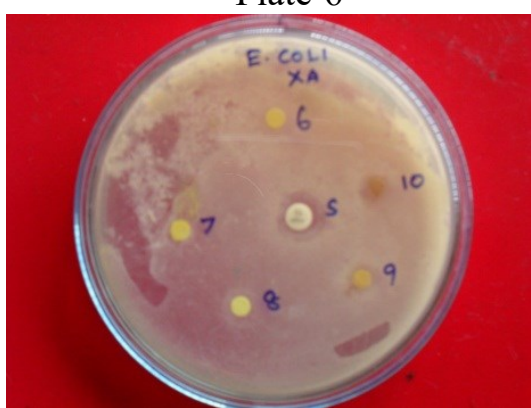

Plate-8

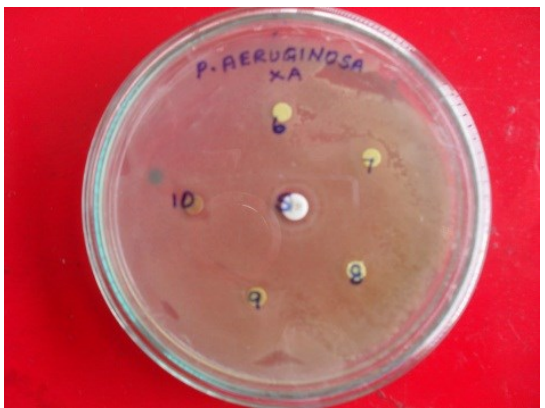

Plate-10

Fig. 5. Antibacterial activity of 3,5-dichloro-2-hydroxyphenyl chalcones (entries 27-36). 


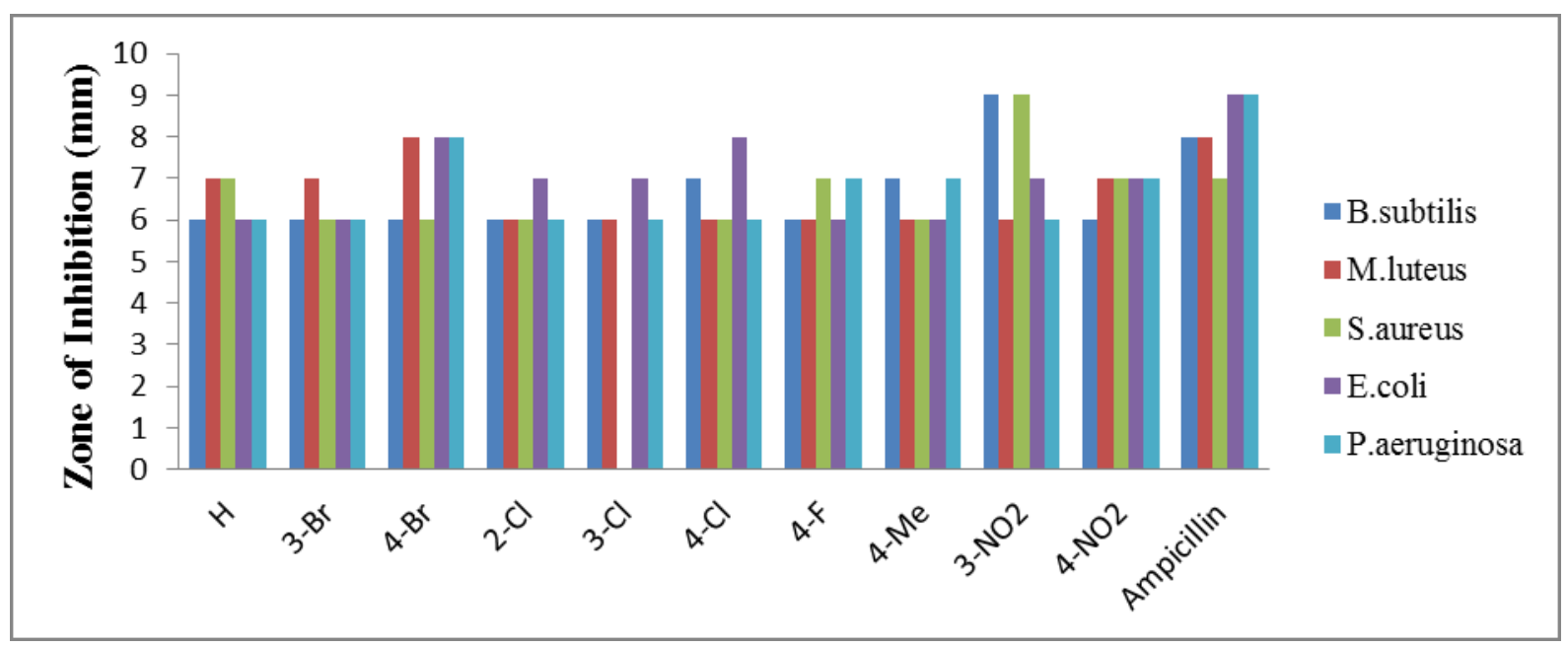

Fig. 6. Antibacterial activity of 3, 5-dichloro-2-hydroxyphenyl chalcones (entries 27-36): clustered column chart.

\section{CONCLUSION}

We have developed an efficient Crossed-Aldol condensation for synthesis of chalcones using thionyl chloride catalyst. The yield of the reaction is more than $80 \%$. The purities of these synthesised chalcones are checked by their physical constants, analytical and spectral data. The spectroscopic data of the chalcones were correlated with Hammett substituent constants, $\mathrm{F}$ and $\mathrm{R}$ parameters. The antibacterial activities of all synthesized chalcones have been studied using Bauer- Kirby method.

\section{Acknowledgement}

The authors thank SAIF, IIT Chennai-600 036, for recording NMR spectra of all compounds.

\section{References}

[1] (a) Thirunarayanan G, Vanangamudi G, Subramanian M, Umadevi U, Sakthinathan S P and Sundararajan R, Elixir Org.Chem, 39 (2011) 4643 - 4653

(b) Ranganathan R, Arulkumaran R, Kamalakkannan D, Vanangamudi G and Thirunarayanan G, IUP J. Chem, 4(2) (2011) 60 - 70

[2] (a) Yankep E, Fomumand Z T, Dangne E. Phytochem, 46 (1997) 591 -593

(b) Sritularak K and Likhitwayawuid, Phytochem, 67 (2006) 812 - 817

[3] Thirunarayanan G and Vanangamudi G E-J. Chem, 4 (1) (2007) 90-97

[4] Ranganathan K, Arulkumaran R, Kamalakkannan D, Sundararajan R, Sakthinathan S P, Vijayakumar S, Suresh R, Vanangamudi G, Thirumurthy K, Mayavel $\mathrm{P}$ and Thirunrayanan G, Int.J. Pharm. Med. and Bio. SC, 1(1) (2012) $62-85$

[5] Venkat Reddy G, Maitraie G, Narsaiah D, Rambahu B, Rao R, Synth. Commun, 31(18), (2001) $2881-2884$ 
[6] Mulliken R S, J. Chem. Phys, 7 (1939) 121-131

[7] Deiva C M, Pappano N B and Debattisata N B, Rev.Microbiol., 29(4) (1998) 307 - 310

[8] Sivakumar M, Phrabusreeneivasan S, Kumarand V, Doble M, Bioorg. Med. Chem. Lett, 17(10) (2000) 3169 - 3172

[9] Lahtchev K L, Batovska D I, St Parushev P, Ubiyvock V M and Sibirny A A, Eur. J. Med. Chem, 43(1) (2008) 2220 - 2228

[10] Weber M W, Hunsaker L A, Abcouwer S F, Decker L M and Vander D L Jagat, Bioorg. Med. Chem, 13 (2005) 3811 - 3820

[11] Parmer V S, Bishit K S, Jain R, Singh S, Sharma S K, Gupta S, Malhotra S, Tyagi O D, Vardhan A, Pati H N, Berghe D V and Vlietinek A J, Indian J. Chem, 35B (1996) 220 224

[12] Dominguez J N, Leon C and Rodrigues J, IL Farmaco., 60(4) (2005), 307 - 311

[13] Arulkumaran R, Sundararajan R, Vanangamudi G, Subramanian M, Ravi K, Sathiyendidran V, Srinivasan S and Thirunarayanan G, IUP J. Chem, 3(1) (2010) 82 98

[14] Lin Y M, Zhon Y, Flavin M T, Zhon L M, Ne W and Chen F C, Bioorg. Med. Chem, 10(8) (2002) $2795-2802$

[15] (a) Liu X and Go M L, Bioorg. Med. Chem, 14 (2006), 153 - 163

(b). Delmulle L, Bellahcene A, Dhooge W, Comhaire F, Roelens F, Huvaere K. Heyerick A, Castronovo V and Keukeleire D D, Phytomed., 13 (2006) 732 - 734

[16] Nielsen S F, Chen M, Theander T G, Kharazmi A and Christensen S B .Bioorg. Med. Chem. Lett, 5 (1995) 449 - 452

[17] Hsieh H K, Tsao L T and Wang J P, J. Pham. Pharmacol, 52 (2000) 163 - 171

[18] Vaiana G S, Banderia M A and Matos F, J. Phytomed., 10 (2003) 189 - 195

[19] (a). Thirunarayanan G, J. Indian Chem. Soc, 84 (2008) 447 - 451

(b). Thirunarayanan G, Surya S, Srinivasan S, Vanangamudi G and Sathyendiran V, Spectrochim Acta., 75A (2010) 152 - 156

[20] Dasharathi D, Netaji R, Basheer M A and Vibhute Y B Ultra Sci, 17(1) (2005) 89 -92

[21] Thirunarayanan G, Mayavel P, Thirumurthy K, Spectrochim Acta, 91A (2012)18-22

[22] Swain C G, Lupton E C Jr., Am. Chem. Soc. 90 (1968) 4328 - 4337

[23] Hays W P, Timmons C J, Spectrochim. Acta, 24(A), (1968) 323- 334

[24] Perjessy A, Hrnciar P, Coll. Czech. Chem. Commun, 35 (1970) 1120 -1128.

[25] Silver N L, Boykin D W, Jr., J. Org. Chem. 35 (1970) 759 - 764

[26] Lautrber P C, J. Am. Chem. Soc, 83 (1961) 1846-1852.

[27] Solcaniova E, Toma S, Fiedlerova A, Org. Mag. Resonance, 14(3) (1980) 181-185.

[28] Bauer A W, Kirby W M, Sherris J C, Truck M, Am. J. Clin. Pathol. 45 (1996) 493498. 